\title{
Neuronal Activity in the Primate Amygdala during Economic Choice
}

\author{
(DAhmad Jezzini ${ }^{1}$ and ${ }^{-C a m i l l o}$ Padoa-Schioppa ${ }^{1,2,3}$ \\ Departments of ${ }^{1}$ Neuroscience, ${ }^{2}$ Economics, and ${ }^{3}$ Biomedical Engineering, Washington University in St. Louis, St. Louis, Missouri 63110
}

Multiple lines of evidence link economic choices to the orbitofrontal cortex (OFC), but other brain regions may contribute to the computation and comparison of economic values. A particularly strong candidate is the basolateral amygdala (BLA). Amygdala lesions impair performance in reinforcer devaluation tasks, suggesting that the BLA contributes to value computation. Furthermore, previous studies of the BLA have found neuronal activity consistent with a value representation. Here, we recorded from the BLA of two male rhesus macaques choosing between different juices. Offered quantities varied from trial to trial, and relative values were inferred from choices. Approximately one-third of BLA cells were task-related. Our analyses revealed the presence of three groups of neurons encoding variables offer value, chosen value, and chosen juice. In this respect, the BLA appeared similar to the OFC. The two areas differed for the proportion of neurons in each group, as the fraction of chosen value cells was significantly higher in the BLA. Importantly, the activity of these neurons reflected the subjective nature of value. Firing rates in the BLA were sustained throughout the trial and maximal after juice delivery. In contrast, firing rates in the OFC were phasic and maximal shortly after offer presentation. Our results suggest that the BLA supports economic choice and reward expectation.

Key words: subjective value; decision making; neurophysiology; neuroeconomics

\section{Significance Statement}

Economic choices rely on the orbitofrontal cortex (OFC), but other brain regions may contribute to this behavior. A strong candidate is the basolateral amygdala (BLA). Previous results are consistent with a neuronal representation of value, but the role of the BLA in economic decisions remains unclear. Here, we recorded from monkeys choosing between juices. Neurons in the BLA encoded three decision variables: offer value, chosen value, and chosen juice. These variables were also identified in the OFC. The two areas differed in the proportion of cells encoding each variable and in the activation timing. In the OFC, firing rates peaked shortly after offer presentation; in the BLA, firing rates were sustained and peaked after juice delivery. These results suggest that the BLA supports choices and reward expectation.

\section{Introduction}

Economic choices entail assigning and comparing the subjective values of different goods. Valuation and decision are thought to involve the orbitofrontal cortex (OFC; Wallis, 2011; PadoaSchioppa and Conen, 2017), but other brain regions may also contribute to these processes. In this respect, the basolateral amygdala (BLA) is a strong candidate. Anatomically, the amygdala is interconnected with prefrontal regions, including orbital and medial prefrontal areas (Porrino et al., 1981; Amaral and Price, 1984;

Received April 29, 2019; revised Nov. 25, 2019; accepted Nov. 27, 2019.

Author contributions: A.J. and C.P.-S. designed research; A.J. performed research; A.J. and C.P.-S. analyzed data; A.J. and C.P.-S. wrote the paper.

This research was supported by the National Institutes of Health (Grant R01-MH104494 to C.P.-S.). We thank Alessandro Livi, Weikang Shi, and Manning Zhang for comments on the manuscript.

The authors declare no competing financial interests.

Correspondence should be addressed to Camillo Padoa-Schioppa at camillo@wustl.edu.

https://doi.org/10.1523/JNEUROSCI.0961-19.2019

Copyright $@ 2020$ the authors
Morecraft et al., 1992; Ghashghaei and Barbas, 2002; Cho et al., 2013). BLA lesions impair performance in goal-directed behavior following reinforcer devaluation (Málková et al., 1997; Blundell et al., 2001; Setlow et al., 2002; Fiuzat et al., 2017; Hart and Izquierdo, 2017), with effects similar to those observed following lesions of the central OFC (Gallagher et al., 1999; Rudebeck et al., 2013). In humans, bilateral amygdala lesions affect gambling and performance in trust games (De Martino et al., 2010; van Honk et al., 2013). In nonhuman primates and rodents, numerous studies have examined BLA activity in the context of reinforcement learning (Ono et al., 1995; Uwano et al., 1995; Parkinson et al., 2001; Setlow et al., 2002; Balleine et al., 2003; Corbit and Balleine, 2005; Chau et al., 2015). For example, Costa et al. (2016) found that amygdala lesions disrupted learning under deterministic and stochastic reinforcement. In addition, BLA neurons were found to encode the valence of positive or negative outcomes, or of outcome-predicting cues (Garavan et al., 2001; Paton et al., 2006; Zhang et al., 2013). Other studies found neuronal activity in the 
a

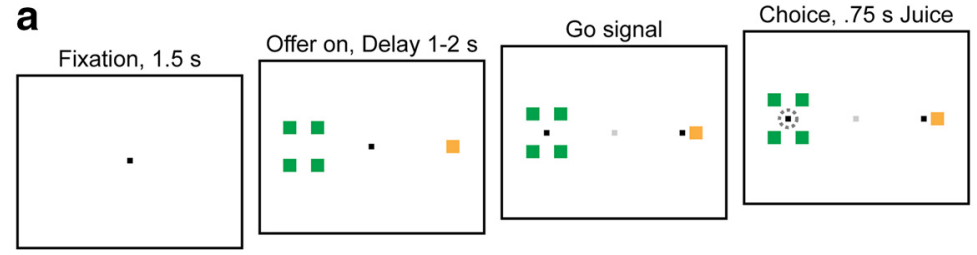

b

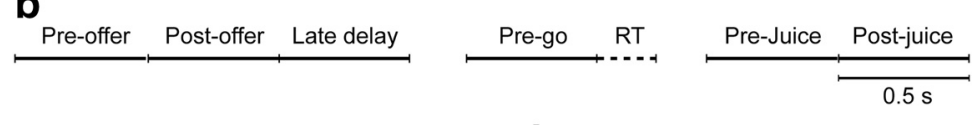

C

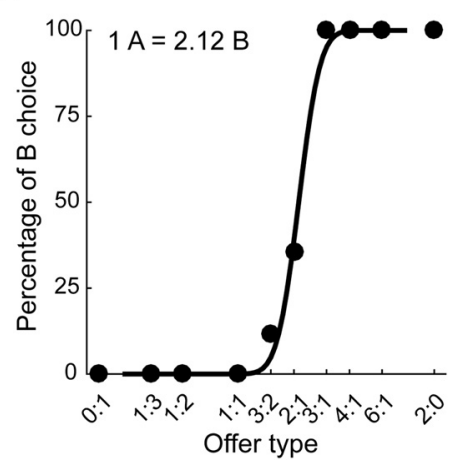

Figure 1. Experimental design and choice patterns. $\boldsymbol{a}$, Task design. At the beginning of the trial, the animal fixated on a central location. Two offers represented by sets of colored squares appeared on the two sides of a fixation point. After a randomly variable delay (1-2s), two saccade targets appeared by the offers (go signal). The animal indicated its choice with a saccade and maintained peripheral fixation for $0.75 \mathrm{~s}$, after which the chosen juice was delivered. $\boldsymbol{b}$, Time windows. $\boldsymbol{c}$, Example choice pattern. The percentage of $B$ choices $\left(y\right.$-axis) is plotted against the $\log$ quantity ratio $\left(\log \left(q_{B} / q_{A}\right), x\right.$-axis). For this session, the probit regression indicated $\rho=2.12$. $\boldsymbol{d}$ MRI scan from monkey $\mathrm{H}$ with the recording electrode placed in the basolateral amygdala. The asterisk indicates the tip of the electrode.

BLA was linearly related to reward magnitude (Bermudez and Schultz, 2010; Jenison et al., 2011) and context adapting (Paton et al., 2006; Saez et al., 2017). Most relevant here, Grabenhorst et al. (2012, 2016) and Zangemeister et al. (2016) showed that BLA responses during a saving-spending task reflected the decision outcome. Together, these results suggest that the BLA might contribute to the mental processes underlying economic choices. However, the behavioral tasks used in most of these studies did not include a choice. Hence, it remains unclear what decision variables may be represented in the BLA during economic decisions, and how the encoding of these variables compares with that found in the OFC.

To shed light on these issues, we recorded from the BLA of macaques engaged in a juice choice task. In this behavioral paradigm, animals choose between two juices offered in variable amounts. In each session, the relative subjective value of the two juices is inferred from the choice pattern and used to interpret neuronal activity. As in previous studies of the OFC (Padoa-Schioppa and Assad, 2006) and anterior cingulate cortex (ACC; Cai and PadoaSchioppa, 2012), we examined the activity of individual cells from the BLA in relation to a large number of variables potentially encoded in this area. Our analyses revealed that neurons in the BLA encoded the identity and values of offered and chosen goods. Specifically, different groups of neurons encoded the value of individual juices (offer value), the binary choice outcome (chosen juice), and the subjective value of the chosen good (chosen value). These results closely resemble those previously obtained for the OFC (Padoa-Schioppa and Assad, 2006). One notable difference between the two areas was that significantly more neurons in the BLA encoded the chosen value, whereas the encoding of offer value and chosen juice was comparatively weaker. In addition, modulations in the BLA were sustained throughout the trial and peaked after juice delivery. In contrast, neuronal activity in the OFC was phasic, peaked shortly after the offer, and presented a secondary peak at juice delivery. Our results suggest that the BLA might participate in the neural processes underlying economic choices. The sustained time course of BLA activation also suggests an interpretation in terms of reward expectation.

\section{Materials and Methods}

All experimental procedures conformed to the NIH Guide for the Care and Use of Laboratory Animals and were approved by the Institutional Animal Care and Use Committee at Washington University in St. Louis.

Experimental procedures. The choice task and the procedures for neuronal recordings were similar to those described in previous studies (Padoa-Schioppa and Assad, 2006; Cai and Padoa-Schioppa, 2012). In brief, two adult male rhesus monkeys (Macaca mulatta; monkey $\mathrm{H}, 10 \mathrm{~kg}$; monkey I, $9 \mathrm{~kg}$ ) participated in the experiments. After a period of chair training and familiarization, monkeys underwent a surgical procedure under general anesthesia to implant a head post and an oval recording chamber (main axes, 30 and $50 \mathrm{~mm}$ ). The chamber was placed on the left hemisphere in monkey $\mathrm{H}$ and on the right hemisphere in monkey I. The chamber was centered on 25 $\mathrm{mm}$ anteroposterior and $\pm 10 \mathrm{~mm}$ mediolateral, with the longer axis parallel to a sagittal plane. Presurgery and postsurgery structural MRIs were used to guide neuronal recordings.

During the experiments, the monkey sat in an electrically insulated enclosure in front of a computer monitor $(57 \mathrm{~cm}$ distance). The gaze direction was monitored with an infrared video camera (EyeLink, SR Research). The choice task was controlled through a custom software (MonkeyLogic) written in MATLAB (MathWorks). In each session, the animals chose between two juices labeled A and B (with A preferred) offered in variable amounts. Figure $1 a$ illustrates the trial structure. At the beginning of each trial, the animal maintained central fixation for $1.5 \mathrm{~s}$. Two offers, represented by sets of color squares, were then presented simultaneously on the two sides of the fixation point. The offers remained on display for a randomly variable delay (1-2s), at the end of which the central fixation point was extinguished (go signal). The animal indicated its choice with a saccade, and after an additional $0.75 \mathrm{~s}$ delay, the chosen offer was delivered. A typical session lasted $\geq 200$ trials. Different pairs of offers were presented pseudorandomly. For each pair of offers, the spatial positions (left/right) were counterbalanced across trials. Across sessions, we used a variety of juice types ( 14 different juices; 25 different juice pairs).

Extracellular recordings were conducted using single tungsten electrodes (FHC; BLA and OFC in monkey $\mathrm{H}$ and OFC in monkey I) or linear arrays (V-probe, Plexon; BLA in monkey I). Electrodes were guided through a custom-made $1 \mathrm{~mm}$ grid. Distances from the top of the grid to the surface of the brain were carefully measured. Postsurgical MRI scans were used to estimate the travel distance necessary to reach the amygdala and $\mathrm{OFC}$, and to reconstruct the recording coordinates. For each animal, we also performed a few dedicated sessions to map the transition between gray and white matter and to register these measures with those obtained from MRI scans. Recording locations were occasionally confirmed with an MRI scan performed with one electrode in place. Neuronal activity 

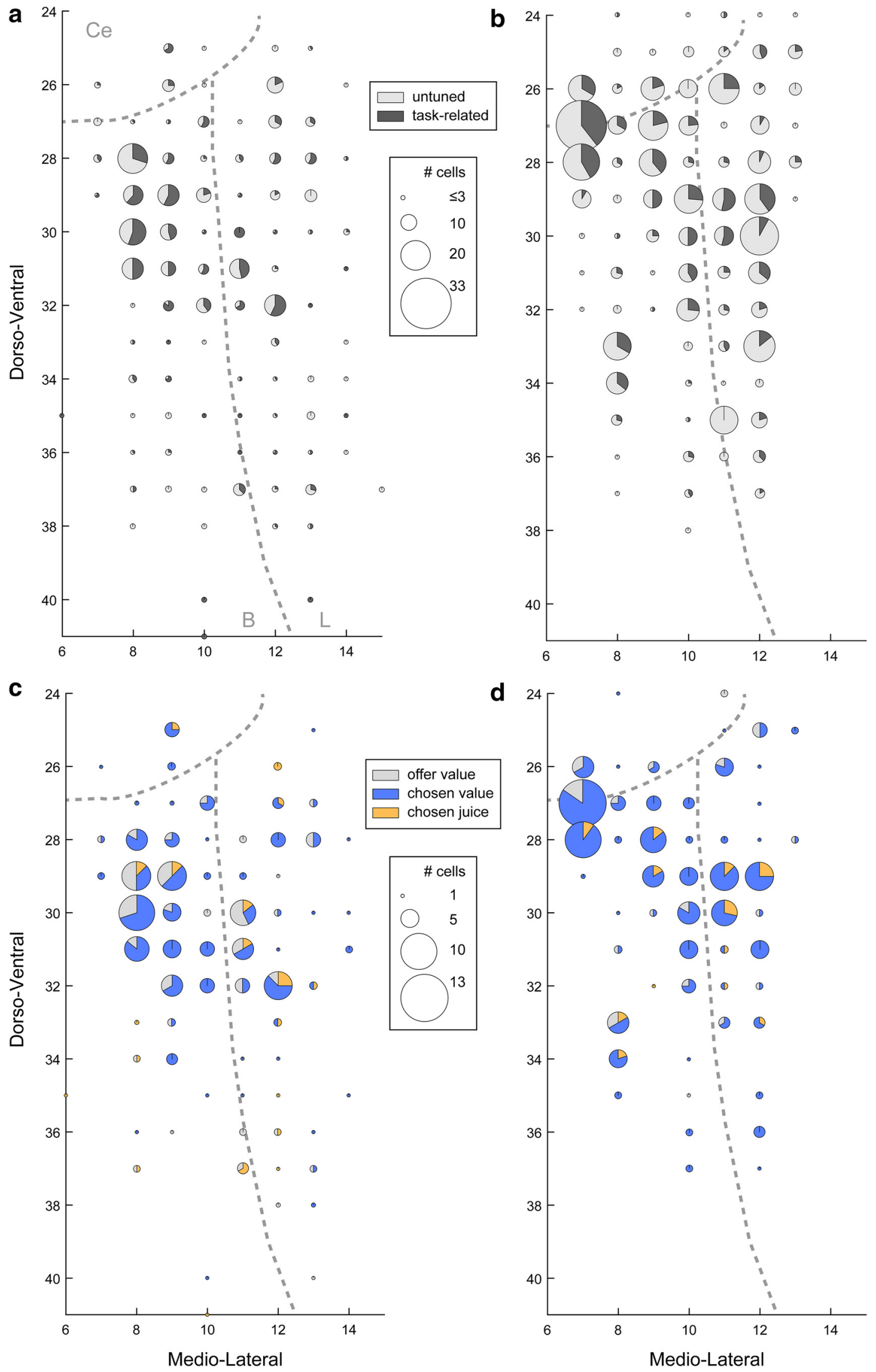

Figure 2. Reconstruction of recording sites. Locations are shown on a $1 \mathrm{~mm}$ grid. The $x$-axis and $y$-axis represent mediolateral and dorsoventral coordinates, respectively. Rostrocaudal locations were collapsed. Mediolateral coordinates are interaural estimates obtained by aligning the recording grid to the postsurgery MRI. Dorsoventral coordinates indicate the depth from the dura (as opposed to interaural coordinates). Dashed lines indicate our best estimate of the boundaries between different nuclei. B, L, and Ce indicate basal, lateral, (Figure legend continues.) 
was amplified, bandpass filtered (300 Hz to $6 \mathrm{kHz}$; Lynx 8, Neuralynx), and acquired at $40 \mathrm{kHz}$ (Power 1401, CED). Spikes were detected online and saved to disk for offline sorting (Spike2, CED). Amygdala recordings were centered on the basolateral nucleus (Fig. 2); OFC recordings were centered on area 13.

Analysis of choice patterns. All analyses were performed in MATLAB (MathWorks). Unless otherwise indicated, the analyses conducted here were as described in previous studies (Padoa-Schioppa and Assad, 2006; Cai and Padoa-Schioppa, 2012).

The quantities of juices $\mathrm{A}$ and $\mathrm{B}$ offered on any given trial are referred to as $q_{\mathrm{A}}$ and $q_{\mathrm{B}}$. In every session, animals presented a quality-quantity trade-off. If the two juices were offered in equal amounts, the animal generally chose juice A (by definition). However, if the $q_{\mathrm{B}} / q_{\mathrm{A}}$ ratio was sufficiently large, the animal chose juice $\mathrm{B}$ consistently. Choice patterns were analyzed with a probit regression, as follows:

$$
\begin{aligned}
& \text { choice } \mathrm{B}=\Phi(X) \\
& X=a_{0}+a_{1} \log \left(q_{\mathrm{B}} / q_{\mathrm{A}}\right),
\end{aligned}
$$

where choice $\mathrm{B}=1$ if $\mathrm{B}$ is chosen and 0 otherwise, and $\Phi(X)$ is the cumulative distribution function of the standard normal distribution. From the fitted parameters, we derived measures for the relative value $\rho=\exp \left(-a_{0} / a_{1}\right)$ and the sigmoid steepness $\eta=a_{1}$. Intuitively, the relative value $\rho$ is the quantity that makes the animal indifferent between $1 \mathrm{~A}$ and $\rho \mathrm{B}$. The steepness $\eta$, also termed inverse temperature, is inversely related to choice variability.

Neuronal classification. Neuronal data were analyzed in seven time windows aligned with different behavioral events: preoffer $(0.5 \mathrm{~s}$ prior the offer onset), postoffer ( $0.5 \mathrm{~s}$ after the offer onset), late delay (from 0.5 to $1 \mathrm{~s}$ after the offer onset), prego ( $0.5 \mathrm{~s}$ before the go cue), reaction time (from the go cue to the saccade start), prejuice $(0.5 \mathrm{~s}$ before the juice delivery), and postjuice ( $0.5 \mathrm{~s}$ after the juice delivery; Fig. $1 b)$. An "offer type" was defined by two offered quantities (e.g., [1A:2B]); a "trial type" was defined by an offer type and a choice (e.g., [1A:2B, B]). For each cell and each time window, we averaged spike counts across trials for each trial type. A "neuronal response" was defined as the firing rate of one cell in one time window as a function of the trial type. Trial types with fewer than two trials were discarded.

For each neuron and each time window, we conducted a three-way ANOVA (factors: offer type $\times$ position of $A \times$ movement direction). As in previous studies, we set a significance threshold of $p<0.001$. As described below, a large majority of neurons were not affected by spatial factors. We thus conducted a one-way ANOVA (factor: trial type). Responses that passed the significance criterion $(p<0.001)$ were included in subsequent analyses. Neurons that passed this criterion in one or more time windows were referred to as "task-related" (Table 1).

We conducted a series of analyses to identify the decision variables encoded in the BLA. As in previous studies, we defined a large number of variables that neurons could conceivably encode. These included variables associated with a single juice (offer value $A$, offer value $B$, etc.), variables reflecting the subjective value of the chosen and unchosen juices (chosen value, other value, value difference, etc.), variables defined by the quantity as opposed to the value (chosen number, total number, etc.), and a variable defined by the binary choice outcome (chosen juice). In total, we examined 19 variables, which are defined in Table 2. For each neuronal response, we performed a linear regression separately on each variable. We thus obtained the regression slope and the $R^{2}$ value. If the regression slope differed significantly from zero $(p<0.05)$, the variable was said to "explain" the response. If the variable did not explain the response, we set $R^{2}=0$. Any given response could be explained by $>1$ variable. In this case, we identified the variable providing the best expla-

$\leftarrow$

(Figure legend continued.) and central nucleus, respectively. $\boldsymbol{a}, \boldsymbol{b}$, Recording locations for monkey $\mathrm{H}(\boldsymbol{a})$ and monkey I $(\boldsymbol{b})$. For each location, the circle radius represents the number of recorded cells (see legend), and colors indicate the proportions of task-related versus untuned cells. $\boldsymbol{c}, \boldsymbol{d}$, Map of cell types for monkey $\mathrm{H}$ (c) and monkeyI (d). For each location, the circle radius represents the number of task-related cells (see legend). Gray shades indicate the fractions of cells classified as offer value (gray), chosen value (blue), or chosen juice (yellow).
Table 1. Results of the ANOVAs

\begin{tabular}{lccccc}
\hline & \multicolumn{2}{l}{ Three-way ANOVA } & & One-way ANOVA \\
\cline { 2 - 4 } & Offer type & Position of A & Movement direction & & Trial type \\
\hline Preoffer & 2 & 0 & 0 & 2 \\
Postoffer & 178 & 16 & 41 & 163 \\
Late delay & 161 & 13 & 31 & 158 \\
Prego & 144 & 8 & 24 & 145 \\
Reaction time & 71 & 2 & 17 & 60 \\
Prejuice & 187 & 9 & 51 & 181 \\
Postjuice & 153 & 2 & 13 & 156 \\
At least 1 & 409 & 29 & 111 & 398 \\
Total & 896 & 50 & 177 & 865 \\
\hline
\end{tabular}

For each neuron and each time window, we conducted a three-way ANOVA (factors: offer type $\times$ position of A $\times$ movement direction) and a one-way ANOVA (factor: trial type). Significance threshold was $p<0.001$ for both tests. Numbers in the table indicate the number of cells passing the significance test.

Table 2. Defined variables

\begin{tabular}{lll}
\hline & Variable name & Definition \\
\hline 1 & Offer value $A$ & Value of juice $A$ offered \\
2 & Offer value $B$ & Value of juice $B$ offered \\
3 & Chosen value & Value of the chosen juice \\
4 & Other value & Value of the nonchosen juice \\
5 & Total value & Chosen value + other value \\
6 & (Chosen-other) value & Chosen value - other value \\
7 & (Other/chosen) value & (Other value)/(chosen value) \\
8 & Total number & Max number + min number \\
9 & Max number & Maximal offered number \\
10 & Chosen number & Chosen number \\
11 & Min number & Minimal offered number \\
12 & Other number & Nonchosen number \\
13 & (Max-min) number & Max number - min number \\
14 & (Chosen-other) number & Chosen number - other number \\
15 & (Min/max) number & (Minimum number)/(maximum number) \\
16 & (Other/chosen) number & (Other number)/(chosen number) \\
17 & Chosen value $A$ & Value of juice A chosen \\
18 & Chosen value B & Value of juice B chosen \\
19 & Chosen juice & Binary: 1 if A chosen and 0 if B chosen \\
\hline
\end{tabular}

nation (highest $R^{2}$ value). For some analyses, we collapsed variables offer value $A$ and offer value $B$ (into variable offer value $A \mid B$ ) and variables chosen value $A$ and chosen value $B$ (into variable chosen value $A \mid B$ ). The collapsed variable was deemed to explain a given response if at least one of the two original variables explained the response. In this case, the collapsed variables was assigned the highest of the two $R^{2}$ values obtained for the original variables. If neither variable explained the response, the collapsed variable was assigned $R^{2}=0$.

To identify a small number of variables that best accounted for the whole population, we used two procedures for variable selection-namely, stepwise and best-subset. Both procedures were originally designed for multilinear regressions in the presence of multicollinearity (Dunn and Clark, 1987; Glantz and Slinker, 2001) and were adapted for the analysis of neuronal populations encoding different variables (Padoa-Schioppa and Assad, 2006). The stepwise procedure is iterative. In the first run, the variable explaining the largest number of responses per time window is selected and all of the responses explained by that variable are removed from the dataset. The procedure is then repeated on the residual dataset until newly selected variables explain less than a minimum percentage of responses. In this study, we set the minimum at $2 \%$. (In other studies, we used $5 \%$ or $1 \%$; here, we found that results were most stable when we imposed the $2 \%$ criterion.)

Importantly, the stepwise procedure does not guarantee optimality. In contrast, the best-subset procedure is exhaustive. For $k=1,2,3, \ldots$, the procedure examines all subsets of $k$ variables, computes the number of responses explained by each subset, and identifies the subset that explains the maximum number of responses. A variant of this analysis examines the total $R^{2}$ value explained across responses for each subset. For each $k$, the explanatory power of the variables selected by the best-subset pro- 
Table 3. Post hoc analysis

\begin{tabular}{|c|c|c|c|c|c|c|c|}
\hline Variable X & Variable $Y$ & $n X$ & $n Y$ & $p$ & $R_{x}^{2}$ & $R_{Y}^{2}$ & $p$ \\
\hline Chosen value & Total value & 29 & 13 & 0.004 & 49.1 & 15.6 & $<10^{-5}$ \\
\hline Chosen value & Value difference & 48 & 6 & 0 & 86.8 & 6.1 & 0 \\
\hline Chosen value & Chosen number & 31 & 4 & $<10^{-7}$ & 61.0 & 12.7 & $<10^{-8}$ \\
\hline Chosen value & Chosen value $A \mid B$ & 219 & 10 & 0 & 216.4 & 9.7 & 0 \\
\hline Offer value $A \mid B$ & Chosen value $A \mid B$ & 26 & 10 & 0.002 & 19.8 & 9.7 & 0.031 \\
\hline Chosen juice & Chosen value $A \mid B$ & 9 & 10 & 0.5 & 10.3 & 9.7 & 0.450 \\
\hline Chosen juice & Chosen value $A$ & 19 & 8 & 0.01 & 15.8 & 5.9 & 0.017 \\
\hline Chosen juice & Chosen value $B$ & 18 & 2 & $<10^{-5}$ & 16.5 & 3.8 & 0.002 \\
\hline
\end{tabular}

We tested the three variables selected by the best-subset method (chosen value, offer value, and chosen juice; variable $X$ ) against highly correlated and presumably competitive alternative variables (variables $\eta$ ). The third and fourth column indicate the number of responses explained only by the variable $X(n X)$ and the number of responses only explained by the variable $Y(n Y)$, respectively. The sixth and seventh columns indicate the total $R^{2}$ values of responses only explained by the variable $X\left(R^{2} X\right)$ and the number of responses only explained by the variable $Y\left(R^{2} \gamma\right)$, respectively. All $p$ values (fifth and last columns) are from binomial tests.

cedure was higher than that of all the other subsets. To establish whether this inequality was statistically significant, we conducted a post hoc analysis. For each selected variable (variable $X$ ), we assessed whether the marginal explanatory power was statistically higher than that of other nonselected variables (variable $Y$ ). For each pair of variables (Table 3 ), $n X$ was the number of responses explained by $X$, not explained by $Y$, and not explained by other selected variables; $n Y$ was the number of responses explained by $Y$ and not explained by either $X$ or other selected variables. We then performed a binomial test to examine whether the inequality $n X>n Y$ was statistically significant. We repeated the same procedure comparing the total $R^{2}$ values of the responses exclusively explained by variable $X$ or $Y$.

After we identified the variables encoded in the amygdala, we assigned each neuronal response to the variable providing the highest $R^{2}$ value. To assess whether this neuronal representation was categorical in nature, we examined the $R^{2}$ value provided by the linear regressions. Consider two variables, $X$ and $Y$. For each response, we computed the difference $\Delta R^{2}=$ $R_{X}^{2}-R_{Y}^{2}$. We then examined the distribution of $\Delta R^{2}$ across the population of responses classified as encoding one of these two variables. A bimodal distribution suggests categorical representation (Padoa-Schioppa, 2013). Bimodality was tested with Hartigan's dip test. A recently developed clustering procedure (Onken et al., 2019) could not be applied due to high session-to-session variability in offer types and choices.

To classify neurons (as opposed to responses), we proceeded as follows. First, we focused on selected variables. Second, for each variable and each time window, we calculated the signed $R^{2}$ value where the sign was that of the regression slope. Third, for each variable, we computed the $\operatorname{sum}\left(R^{2}\right)$ as the total $R^{2}$ value across time windows. The encoded variable was that providing the highest $\left\|\operatorname{sum}\left(R^{2}\right)\right\|$, and the sign of the encoding was that of $\operatorname{sum}\left(R^{2}\right)$.

Analysis of $U$-shaped responses. Many neurons in the BLA encoded the chosen value. We examined whether their firing rates reflected the subjective nature of value. This analysis closely resembled that conducted in previous studies (Padoa-Schioppa and Assad, 2006; Cai and PadoaSchioppa, 2012).

For each response in the dataset, we separated trials in which the animal chose juice A or juice B. For each group of trials, we regressed firing rates on the amount of juice chosen by the animal (linear fit). We thus obtained two slopes, $\beta_{\mathrm{A}}$ for juice $\mathrm{A}$ and $\beta_{\mathrm{B}}$ for juice $\mathrm{B}$. If both slopes differed significantly from zero $(p<0.01)$ and had the same sign, the response was said to be "U-shaped." Importantly, this procedure identified U-shaped responses in an unbiased way.

If $\mathrm{U}$-shaped responses encode the chosen value, the slope ratio $\beta_{\mathrm{A}} / \beta_{\mathrm{B}}$ should provide a neuronal measure for the relative value of the two juices $\left(\rho_{\text {neuronal }}=\beta_{\mathrm{A}} / \beta_{\mathrm{B}}\right)$. Furthermore, this measure should reflect the subjective nature of value and should be indistinguishable from the behavioral measure obtained from the choice pattern $\left(\rho_{\text {behavioral }} \equiv \rho\right.$; see above). To test this prediction, we took advantage of the fact that, for any given juice pair, $\rho_{\text {behavioral }}$ varied to some extent from session to session. We thus performed an ANCOVA using the neuronal measure $\log \left(\rho_{\text {neuronal }}\right)$ as a dependent variable, the behavioral measure $\log \left(\rho_{\text {behavioral }}\right)$ as a predictor, and grouping data by the juice pair. Data recorded from different monkeys and with different juice pairs were grouped separately because relative values generally depend on the subject and on the juice pair. We used the full ANCOVA model, and we included only juice pairs with $\geq 10$ U-shaped responses.

\section{Results}

Two monkeys performed a juice choice task (Fig. $1 a, b$ ). In each session, the animal chose between two juices labeled A and B, with A preferred. Offers were represented by two sets of colored squares, and the monkey indicated its choice with a saccade. The offered quantities and the spatial configuration of the offers varied pseudorandomly. Choice patterns presented a quality-quantity trade-off (Fig. 1c). Measures for the relative value of the juices $(\rho)$ and for the sigmoid steepness $(\eta)$ were derived from a probit regression (see Materials and Methods; Eq. 1).

\section{Neuronal encoding of decision variables in the amygdala}

We recorded a total of 1132 neurons from the BLA ( 432 cells from monkey H, 700 cells from monkey I; Figs. 1d, 2). We analyzed firing rates in seven time windows aligned with different behavioral events (see Materials and Methods). A "neuronal response" was defined as the activity of one cell in one time window.

Visual inspection of individual responses revealed several interesting facts. First, a sizeable fraction of neurons appeared modulated by the offer type. In many cases, neuronal responses seemed to encode the variable chosen value. Figure $3 a$ illustrates one example. This response, plotted against the offer type, had a characteristic $U$ shape (dictated by the offer types included in the experiment). Plotting the firing rate against the variable chosen value revealed a close-to-linear relation. Figure $3 b$ illustrates another example. In this case, the variable chosen value was encoded with a negative slope. Compared with previous findings for the OFC, responses encoding the chosen value were very prevalent in the amygdala. However, we also observed neurons encoding other variables. For example, the activity of the cell in Figure $3 c$ increased as a function of the quantity of juice A offered and did not vary with the quantity of juice $B$. Plotting the firing rate against the variable offer value A revealed a close-to-linear relation. Similarly, the response in Figure $3 d$ seemed to encode the variable offer value $B$. Figure $3 e$ illustrates another example. In this case, firing rates were close to binary- high when the animal chose juice $A$ and low when it chose juice B. Thus, this response seemed to encode the variable chosen juice.

For a quantitative analysis, we proceeded in steps. First, for each cell and each time window, we conducted a three-way ANOVA (factors: offer type $\times$ position of $A \times$ movement direction), and we imposed a significance threshold of $p<0.001$. As detailed in Table 1, 409 of 1132 (36\%) cells were significantly modulated by the offer type in at least one time window. In contrast, fewer cells were modulated by the spatial configuration of the offers $(29 / 1132=3 \%)$ or by the movement direction $(111 /$ 

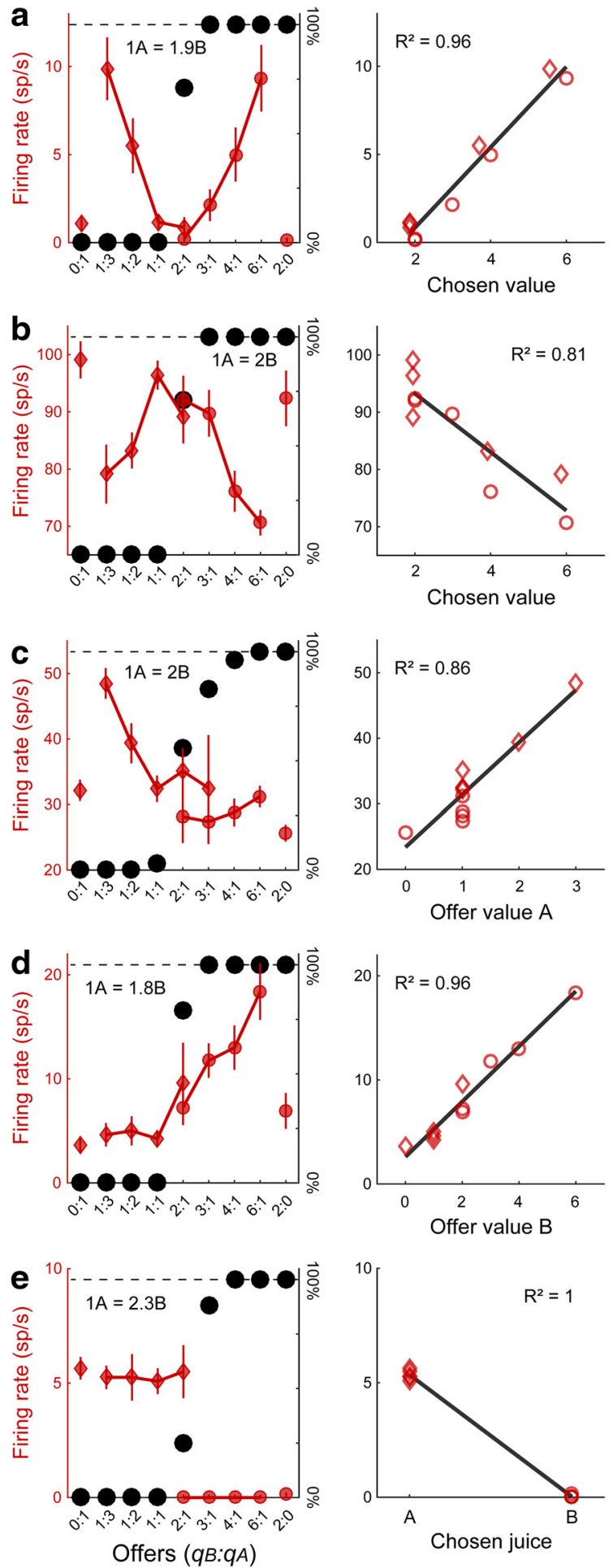

Figure 3. Example of neuronal responses. $\boldsymbol{a}$, Response encoding the chosen value. Left, Black dots indicate the choice pattern and red symbols indicate firing rates. Diamonds and circles indicate trials in which the animal chose juice A and juice B, respectively. Right, The same neuronal response is plotted against the variable chosen value. The black line is from a linear regression ( $R^{2}$ is indicated). $\boldsymbol{b}$, Response encoding the chosen value (negative encoding). In this case, the firing rate of the cell decreased as a function of the chosen value. $c$, Response encoding offer value A. $\boldsymbol{d}$, Response encoding offer value B. $\boldsymbol{e}$, Response encoding the chosen juice. $\boldsymbol{b}$ - $\boldsymbol{d}$, Conventions are as in $\boldsymbol{a} . \boldsymbol{a}-\boldsymbol{e}$, Responses are from the following time windows: postoffer $(\boldsymbol{a})$, prego (b), postjuice (c), postoffer (d), postjuice (e).
$1132=10 \%$ ). We then conducted a one-way ANOVA (factor: trial type). When all time windows were pooled (except the preoffer), 863 neuronal responses from 398 neurons passed the statistical criterion $(p<0.001$; Table 1). Subsequent analyses focused on this population.

As in previous studies, we defined a large number of candidate variables potentially encoded by the population. These included variables associated with a single juice (offer value $A$, offer value $B$, etc.), a variable reflecting the binary choice outcome (chosen juice), variables reflecting the relative value of the two juices (chosen value, other value, etc.), and variables reflecting the numerosity (chosen number, total number, etc.; 19 variables total; see Materials and Methods and Table 2). For each response, we performed a linear regression on each variable, and we obtained the slope and the $R^{2}$ value. If the slope differed significantly from zero $(p<0.05)$, the variable was said to "explain" the response. Figure 4 illustrates the results obtained for the whole population. For each time window, Figure $4 a$ indicates the number of cells explained by each variable. In this plot, each response may contribute to $>1$ bin (because any response could be explained by $>1$ variable). In contrast, Figure $4 b$ indicates the number of responses for which any given variable provided the best explanation (highest $R^{2}$ value). Thus, in this plot, each response contributes to $\leq 1$ bin. Inspection of Figure $4 b$ reveals that the variable chosen value was the most dominant. In addition, smaller populations of responses were best explained by variables offer value $A \mid B$, chosen value $A \mid B$, or chosen juice.

Collectively, the 19 variables examined in our analysis explained 804 of 863 (93\%) responses passing the ANOVA. To identify a small subset of variables that could best account for the whole population, we conducted a stepwise analysis and a bestsubset analysis (see Materials and Methods). In the first iteration, the stepwise procedure selected the variable chosen value, which explained 605 of $863(70 \%)$ responses (Fig. $5 a, b)$. Once these responses were removed, the residual dataset was almost exclusively concentrated on variables offer value $A \mid B$, chosen value $A \mid B$, and chosen juice (Fig. 5a). In the second and third iterations, the stepwise procedure selected variables offer value $A \mid B$ and chosen juice. The three selected variables collectively explained 759 responses, corresponding to $88 \%$ of task-related responses and $94 \%$ of responses explained by all 19 variables. Variables selected in subsequent iterations did not reach the $2 \%$ criterion (Fig. $5 a, b)$. Note that because offer value $A \mid B$ and chosen juice were both correlated with chosen value $A \mid B$, once the former two variables were selected, most of the responses best explained by the latter variable were removed from the dataset (Fig. 5a).

Importantly, the stepwise procedure is path-dependent and does not guarantee optimality. We thus conducted a best-subset analysis (see Materials and Methods). In one variant of this analysis, variables were selected for the maximum number of responses explained. By this criterion, the best subset of three variables included chosen value, offer value $A \mid B$, and chosen value $A \mid B$. In another variant of the analysis, variables were selected for the maximum total $R^{2}$ value (see Materials and Methods). By this criterion, the best subset of three variables included chosen value, offer value $A \mid B$, and chosen juice (Fig. $5 c$ ). To gauge the significance of these observations, we conducted a post hoc analysis comparing the marginal explanatory power of each variable in the best subset with that of other, nonselected variables (see Materials and Methods; Table 3). The explanatory power of chosen value was significantly higher than that of any competing variable (all $p<0.005$ ). The explanatory power of offer value $A \mid B$ was significantly higher than that of chosen value $A \mid B$, both for the 

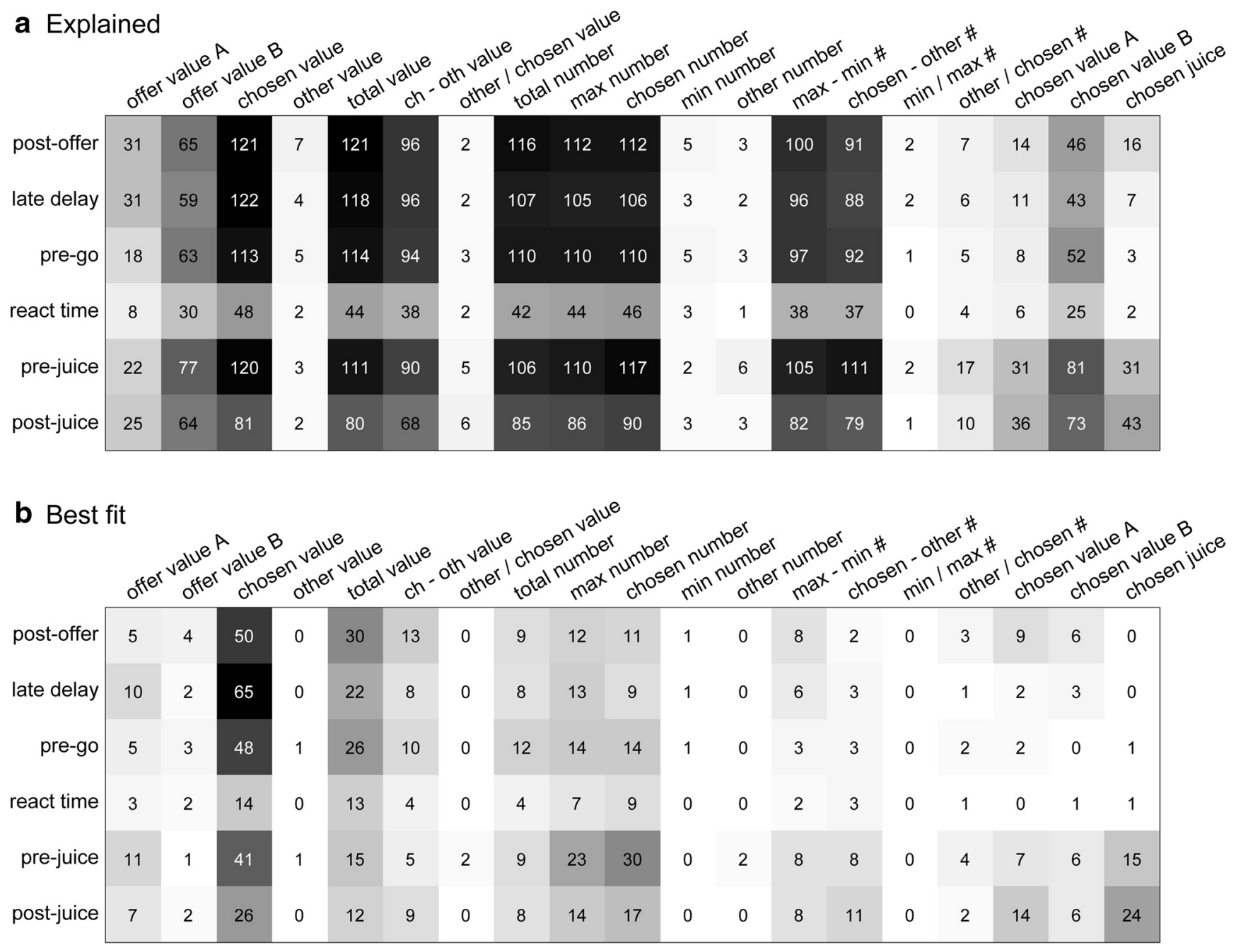

Figure 4. Population results of the linear regression analysis. $a$, Explained responses. Each number indicates the number of responses explained by one variable in one time window. For example, in the postoffer time window, the variable chosen value explained 121 responses. Of note, because variable chosen value is highly correlated with other variables (total value, chosen number, etc.), many of these responses contributed to multiple bins in this plot. Different shades of gray represent the same results indicated numerically. $\boldsymbol{b}$, Best fit. Here, numbers indicate the number of responses for which the corresponding variable provided the best fit (highest $R^{2}$ value). In this panel, each response appears in one bin at most. Here, the variable chosen value clearly dominates across time windows.

number of responses explained ( $p=0.002$ ) and for the total $R^{2}$ value $(p=0.03)$. In contrast, the explanatory power of chosen juice was statistically indistinguishable from that of chosen value $A \mid B$, both for the number of responses explained $(p=0.5)$ and for the total $R^{2}$ value $(p=0.45)$.

These results indicated unequivocally that neurons in the amygdala encoded variables chosen value and offer value $A \mid B$. Conversely, they did not fully disambiguate between variables chosen juice and chosen value $A \mid B$. However, we noted that the latter is a collapsed variable (see Table 2) and thus has a statistical advantage. Conversely, the explanatory power of variable chosen juice was significantly higher than that of either variable chosen value $A$ or chosen value $B$ taken alone (Table 3, bottom rows). Furthermore, the present study aimed to contrast the results obtained for the amygdala with those obtained for the OFC, and we intended to be cautious in identifying differences between areas. Thus, we provisionally concluded in favor of the variable chosen juice. We classified each neuronal response in the population as encoding one of the three selected variables (offer value $A \mid B$, chosen value, and chosen juice). Each response was assigned to the variable providing the highest $R^{2}$ value. We thus identified 165 offer value $A \mid B$ responses, 588 chosen value responses, and 110 chosen juice responses. Figure $5 d$ illustrates the distribution of $R^{2}$ values obtained for each population.

We further analyzed the nature of this neuronal representation by computing, for each pair of variables and for each relevant response, the difference in $R^{2}$ value obtained for the two variables. We then examined the distribution of $\Delta R^{2}$ (see Materials and Methods). For variables chosen value and offer value $A \mid B$, the distribution of $\Delta R^{2}$ presented a significant dip near zero ( $p=0$; Fig. 6b), indicating that the two groups of responses were categorically distinct. Similarly, for variables chosen value and chosen juice, the distribution of $\Delta R^{2}$ presented a significant dip near zero ( $p=0$; Fig. $6 c$ ). In contrast, for variables offer value $A \mid B$ and chosen juice, the dip in the distribution of $\Delta R^{2}$ did not reach significance ( $p=0.2$; Fig. $6 c$; all $p$ values from Hartigan's dip test).

In summary, our analyses indicated the presence of a large and categorically distinct group of neuronal responses encoding the variable chosen value. Other task-related responses appeared to form smaller and possibly noncategorical clusters centered on variables offer value $A \mid B$ and chosen juice (or possibly chosen value $A \mid B)$. In principle, a larger dataset could 

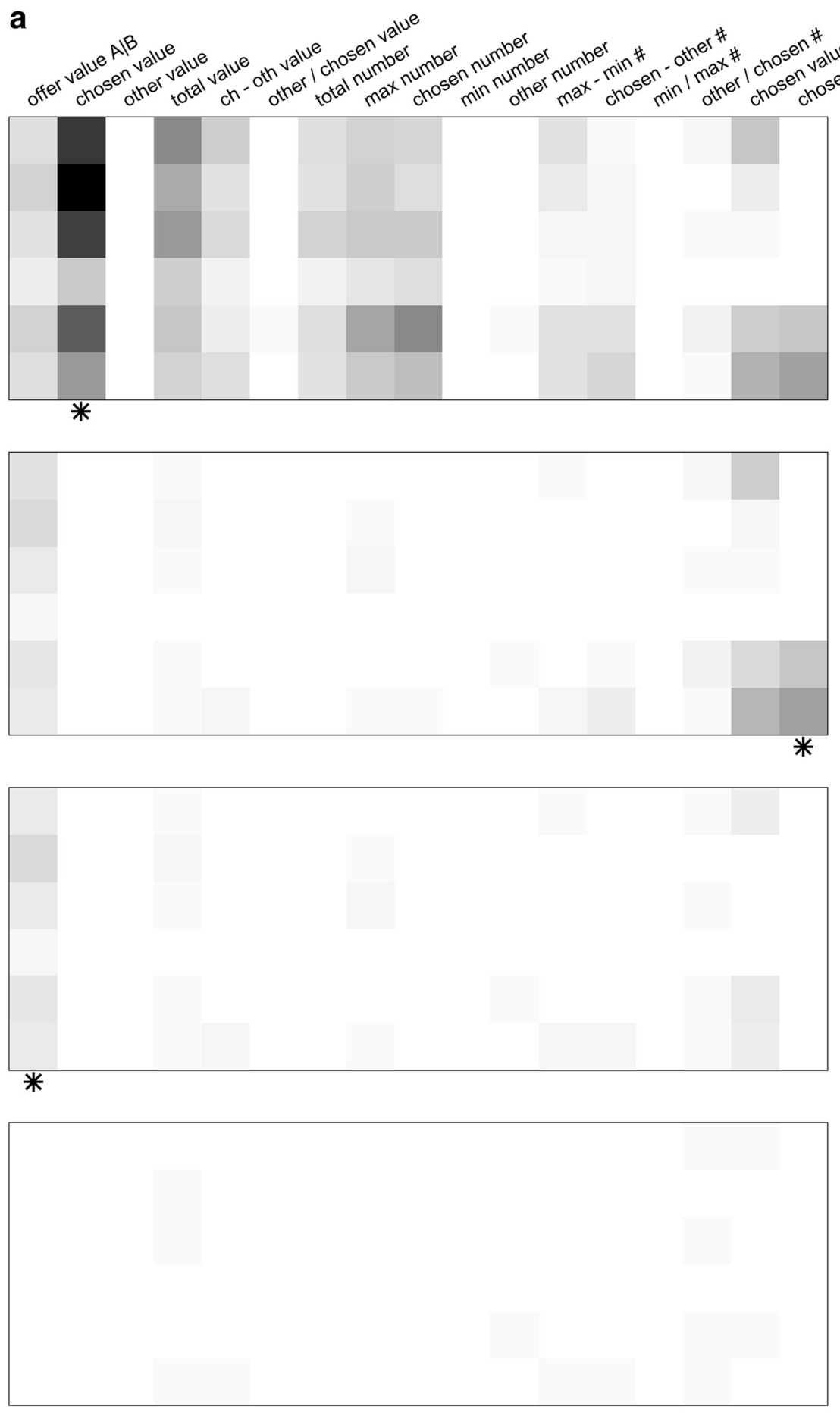
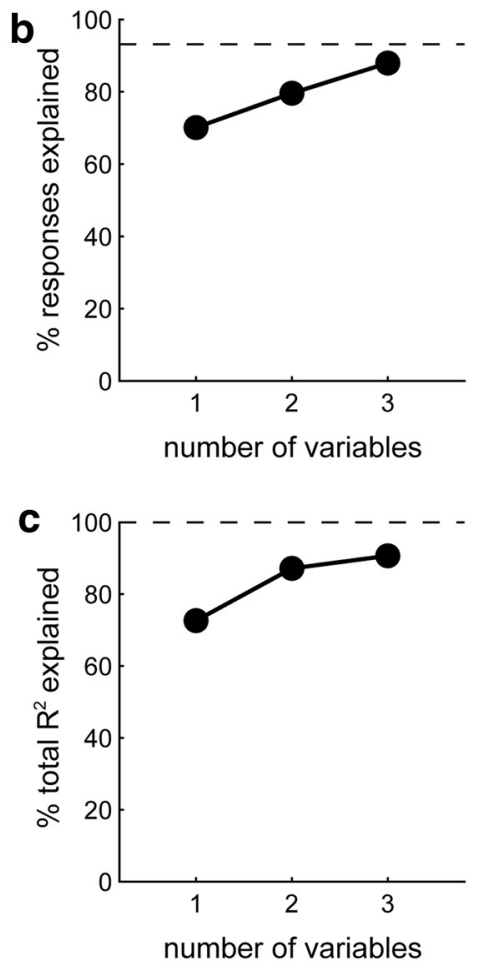

d
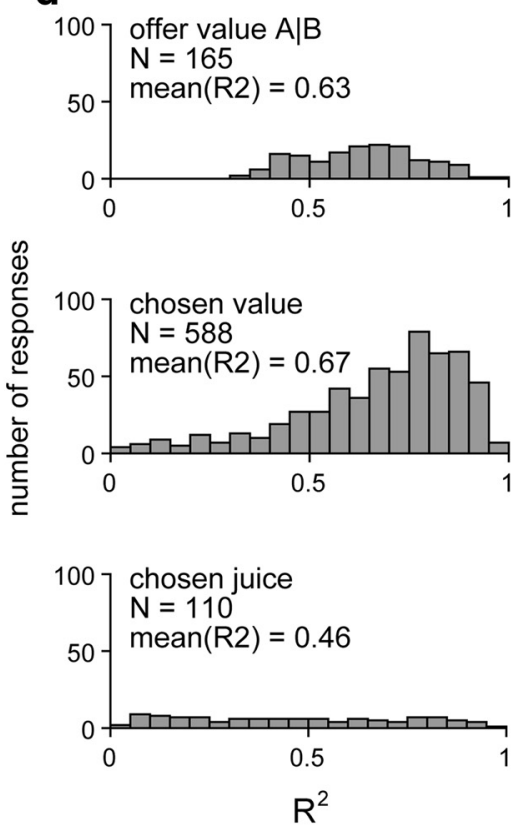

Figure 5. Variable selection analysis. $\boldsymbol{a}$, Stepwise procedure. Top, Same as in Figure $4 b$ (using the same gray scale; see legend of Fig. $4 b$ ). In the first three iterations, the procedure selects the variables chosen value, chosen juice, and offer value $A \mid B$ (asterisk indicates selected variables). Variables selected in subsequent iterations did not reach the $2 \%$ criterion (dot indicates selected-anddiscarded variables). $\boldsymbol{b}$, Percentage of explained responses, stepwise procedure. The panel illustrates the percentage of responses explained as a function of the number of selected variables. Here, $100 \%$ on the $y$-axis represents the total number of task-related responses $(N=863)$. The dotted line indicates the number of responses explained by the 19 variables $(N=804)$. Collectively, the three variables offer value $A \mid B$, chosen value, and chosen juice explained 759 responses. $c$, Total $R^{2}$ value explained, best-subset procedure. The total $R^{2}$ value $(y$-axis) is expressed as a percentage of the theoretical maximum, defined as sum $\left(R_{\text {max }}^{2}\right)$ across the population. For each cell, $R_{\text {max }}^{2}$ is the highest $R^{2}$ value provided by one of the 19 variables. $d$, Distribution of $R^{2}$ values. Each response was classified as encoding one of the variables offer value $A \mid B$, chosen value, and chosen juice. Notably, many more responses encoded the chosen value compared with other variables. Each histogram represents the distribution of $R^{2}$ values for the corresponding variable.

resolve the current ambiguity regarding these smaller clusters (i.e., the encoded variables and the categorical nature of the representation). For the rest of this study, we conservatively classified neuronal responses in three groups defined by vari- ables offer value $A \mid B$, chosen value, and chosen juice. A spatial reconstruction of the recording locations (Fig. 2) did not reveal any clear topographic organization of the different groups of cells within the BLA. 

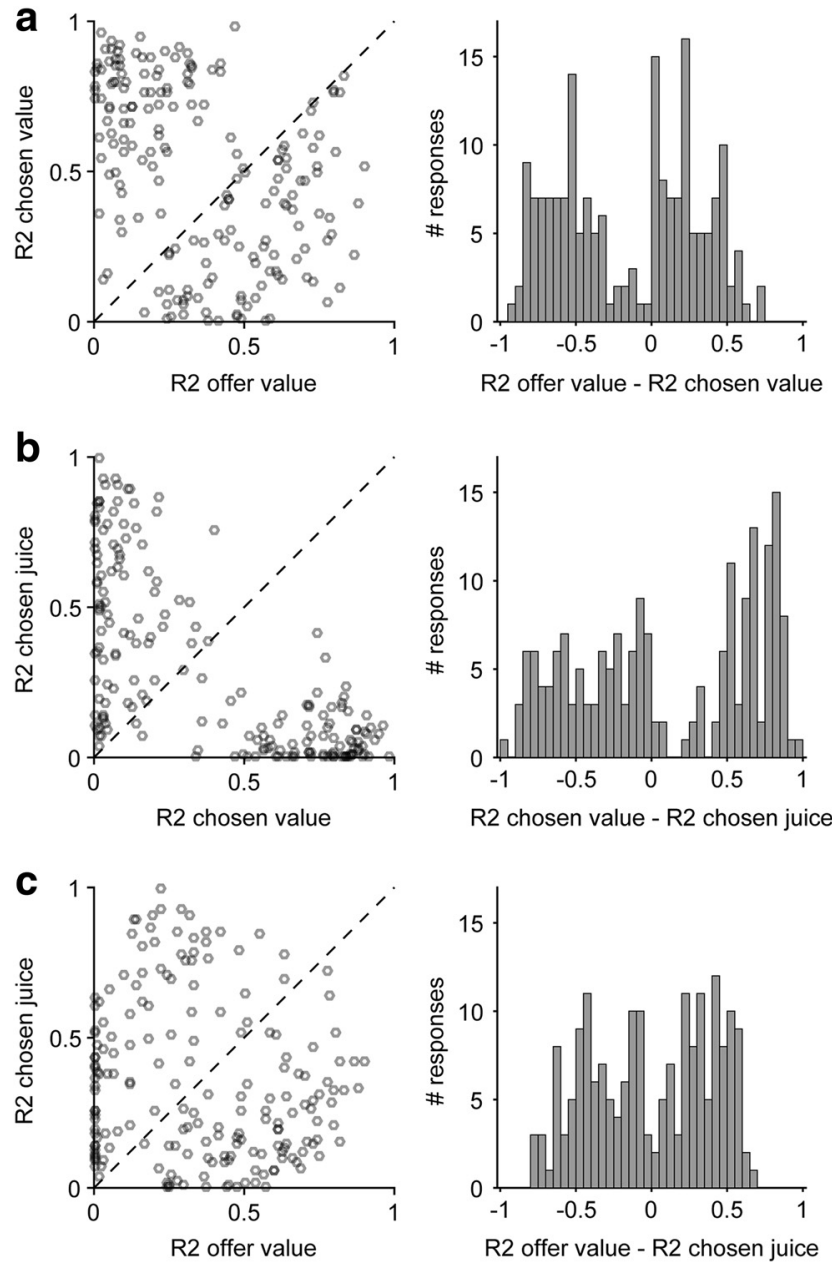

Figure 6. Categorical encoding. For each pair of selected variables, we examined the difference in $R^{2}$ values. $\boldsymbol{a}$, Variables offer value and chosen value. We identified responses encoding offer value $A$ or chosen value. Because many more responses encoded the latter, we randomly down sampled so that the two populations were of equal numbers. For each response, we computed $R_{\text {offer value A }}^{2}$ and $R_{\text {chosen value }}^{2}$. We then repeated these operations for responses encoding the offer value $B$ or the chosen value, and we pooled the results obtained for the two juices $A$ and $B$. We then plotted $R^{2}$ offer value against $R^{2}$ chosen value (left) and the distribution for the difference $\Delta R^{2}$ (right). The distribution for $\Delta R^{2}$ presented a significant dip ( $p=0$, Hartigan's dip test), indicating that neuronal responses encoding variables offer value and chosen value were categorically distinct. $\boldsymbol{b}$, Variables chosen value and chosen juice. The distribution for $\Delta R^{2}$ presented a significant dip ( $p=0$, Hartigan's dip test). c, Variables offer value and chosen juice. Same procedures as for $\boldsymbol{a}$. The dip in the distribution for $\Delta R^{2}$ did not reach the significance threshold ( $p=0.2$, Hartigan's dip test). We repeated these analyses separately for each monkey and obtained very consistent results. For the three pairs of variables, Hartigan's dip test indicated $p=0.001, p=0.001$ and $p=0.2$ for monkey $\mathrm{H}$, and $p=0.024, p=0.001$ and $p=$ 0.29 for monkey l.

\section{$\mathrm{U}$-shaped responses reflect the subjective nature of value}

Many neuronal responses in the amygdala encoded the chosen value. Importantly, this variable reflected the relative value of the juices, which could vary to some extent from session to session. We thus conducted a specific analysis to assess whether variability in chosen value responses matched the variability observed at the behavioral level.

For each response, we examined separately trials in which the animal chose juice A and juice B. For each group of trials, we regressed firing rates against the chosen quantity, and we obtained the regression slopes, $\beta_{\mathrm{A}}$ and $\beta_{\mathrm{B}}$. Responses for which both slopes differed significantly from zero $(p<0.01)$ were identified as U-shaped (see Materials and Methods). Figure $7 a$ illustrates one example. If indeed this response encoded the chosen value, $\beta_{\mathrm{A}}$ should be proportional to the value of juice $A, \beta_{B}$ should be proportional to the value of juice $B$, and the ratio $\beta_{\mathrm{A}} / \beta_{\mathrm{B}}$ should equal the relative value of the two juices. Furthermore, this neuronal measure of relative value $\left(\rho_{\text {neuronal }}=\beta_{\mathrm{A}} / \beta_{\mathrm{B}}\right)$ should be indistinguishable from the corresponding measure obtained from behavioral choices through the probit regression $\left(\rho_{\text {behavioral }}\right)$.

For the response in Figure $7 a$, we measured $\rho_{\text {behavioral }}=2.2$ and $\rho_{\text {neuronal }}=2.3 \pm 1.0$, consistent with the prediction. In itself, this observation did not rule out that this cell encoded some physical property of the juices, such as the sugar content. The key question was whether session-to-session variability in $\rho_{\text {behavioral }}$ was matched by variability in $\rho_{\text {neuronal }}$. To address this issue, we considered the whole population of U-shaped responses. We performed an ANCOVA (full model) regressing $\log \left(\rho_{\text {neuronal }}\right)$ on $\log \left(\rho_{\text {behavioral }}\right)$ and grouping responses by the monkey and by the juice pair (Fig. $7 b$ ). We imposed that $\geq 10$ data points be available for each juice pair. The analysis showed that the neuronal measure, $\log \left(\rho_{\text {neuronal }}\right)$, depended significantly on the behavioral measure, $\log \left(\rho_{\text {behavioral }}\right)(p=0.0004)$. Furthermore, an examination of the slopes and intercepts obtained for each juice pair revealed that, in most cases, the relation between $\log \left(\rho_{\text {neuronal }}\right)$ and $\log \left(\rho_{\text {behavioral }}\right)$ was statistically indistinguishable from identity (Fig. 7c).

The results illustrated in Figure 7 demonstrate that U-shaped responses in the amygdala reflected the subjective nature of value. Indeed, if these neurons encoded any physical property of the juices, the ratio $\beta_{\mathrm{A}} / \beta_{\mathrm{B}}$ would be constant and would not covary with the behaviorally measured relative value of the two juices. In other words, regression lines in Figure $7 b$ would be horizontal. Session-to-session fluctuations in $\rho_{\text {behavioral }}$ can be seen as forms of natural devaluation and revaluation. The fact that the relation between $\rho_{\text {neuronal }}$ and $\rho_{\text {behavioral }}$ is indistinguishable from identity means that U-shaped responses incorporate this internal variability.

\section{Activity profiles and comparison with $\mathrm{OFC}$}

We next examined the time course of activation for neurons encoding the variables identified in the amygdala. Each cell was classified according to the $\operatorname{sum}\left(R^{2}\right)$ across time windows. We pooled offer value cells associated with the two juices and chosen juice cells associated with the two juices. For both groups of cells, we refer to the encoded juice as "juice E" and to the other juice as "juice O." For chosen juice cells, we defined juice $\mathrm{E}$ as that eliciting higher firing rates. Thus, our dataset included 55/16 offer value cells with positive/ negative encoding, 214/38 chosen value cells with positive/negative encoding, and 35 chosen juice cells. We examined the activity profiles of these populations over the course of the trial.

For each chosen value cell, we divided trials into tertiles according to the chosen value (low, medium, and high), and we computed the activity profile for each tertile. We then averaged the profiles across neurons for each population, separately for positive and negative encoding. Focusing on cells with positive encoding (Fig. $8 a$ ), we noted that their activity became significantly modulated by the chosen value $\sim 200 \mathrm{~ms}$ after offer presentation. This modulation remained sustained throughout the delay and after the go signal, and the activity further increased shortly before juice delivery. Similarly, the activity of cells with negative encoding (Fig. $8 b$ ) became modulated by the chosen value within $250 \mathrm{~ms}$ after the offer. This modulation remained sustained throughout the trial until after juice delivery.

For offer value cells, we divided trials into tertiles according to the offer value of juice E. Focusing on the population with positive encoding (Fig. 8 c), we found that the activity became signif- 

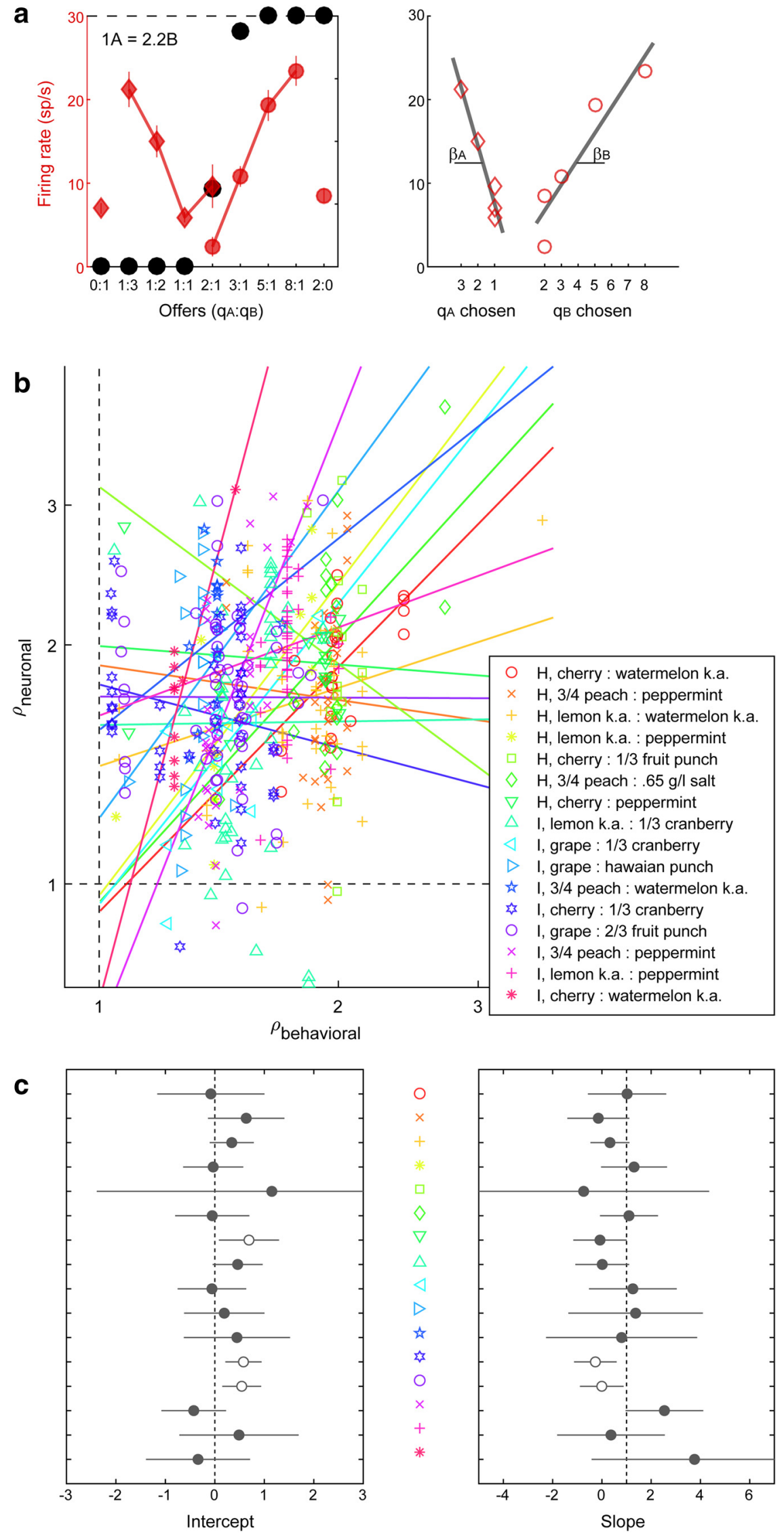

Figure 7. U-shaped responses reflect the subjective nature of value. $\boldsymbol{a}$, Example response. Left, Conventions are as in Figure $3 a$. Right, Firing rates were regressed on the chosen juice quantity, separately for trial types in which the animal chose juice $A$ and juice B. We thus obtained the two slopes $\beta_{A}$ and $\beta_{B}$. The slope ratio provided a neuronal measure for the relative value of the juices icantly modulated by the encoded variables $\sim 200 \mathrm{~ms}$ after offer presentation. This modulation persisted throughout the delay, after the go signal, until after juice delivery. (The population of offer value cells with negative encoding was too small, resulting in noisy activity profiles, and was not analyzed further in this study.)

For chosen juice cells, we divided trials according to the chosen juice. Inspection of the activity profiles (Fig. $8 d$ ) reveals that this population reflected the choice outcome (i.e., the chosen juice) within 250 $\mathrm{ms}$ of the offer. These neurons maintained a sustained modulation throughout the delay, and this modulation further increased upon juice delivery.

The time profiles observed in Figure $8 a-c$ appeared somewhat different from what was observed in previous studies of the OFC, where average firing rates were lower, and where offer value and chosen value cells presented more transient modulations (Padoa-Schioppa, 2013). Importantly, previous studies of the OFC were conducted in different animals. To confirm that differences in activity profiles revealed differences between brain regions as opposed to differences between individual animals, we collected a new dataset from the OFC. Specifically, we recorded the activity of 716 neurons (364 from monkey H; 352 from monkey I). Using the same procedures described above and in previous studies, we identified taskrelated responses with an ANOVA $(p<$ 0.001 ), and we classified neurons according to the variables characteristic of the

$\leftarrow$

$\rho_{\text {neuronal }}=\beta_{\mathrm{A}} / \beta_{\mathrm{B}}$, which we compared with the behavioral measure obtained from the probit regression. For this response, $\rho_{\text {behavioral }}=2.2$ and $\rho_{\text {neuronal }}=2.3 \pm 1.0$ (mean \pm SEM; SEM calculated through error propagation). $\boldsymbol{b}$, Population analysis. Here, $\rho_{\text {neuronal }}$ and $\rho_{\text {behavioral }}$ are plotted against each other in log-log scale. Each symbol represents one response, different colors indicate different juice pairs (see legend), and regression lines show the results of the ANCOVA (full model). In the legend, $\mathrm{H}$ and I identify the animal. In total, 427 responses from 16 juice pairs were included in the analysis. $\boldsymbol{C}$, Estimates for individual juice pairs. Left, right, The estimate obtained from the ANCOVA for the intercept (left) and for the slope (right), separately for each juice pair. The association between juice pairs and color symbols is defined in $\boldsymbol{b}$. Values on the $x$-axes refer to the relation between $\log \left(\rho_{\text {neuronal }}\right)$ and $\log \left(\rho_{\text {behavioral }}\right)$. Vertical dotted lines (intercept $=0$, slope $=$ 1) correspond to the values predicted by the identity $\rho_{\text {neuronal }}=$ $\rho_{\text {behavioral }}$ Error bars indicate 3 SES, and empty circles highlight juice pairs for which, by this measure, data points differ significantly from the values 0 (intercept) and 1 (slope). For 13 of 16 pairs, the relation between $\rho_{\text {neuronal }}$ and $\rho_{\text {behavioral }}$ was indistinguishable from identity. Note that very similar results were obtained for the two animals. 
a chosen value, slope $>0$ (214 cells)

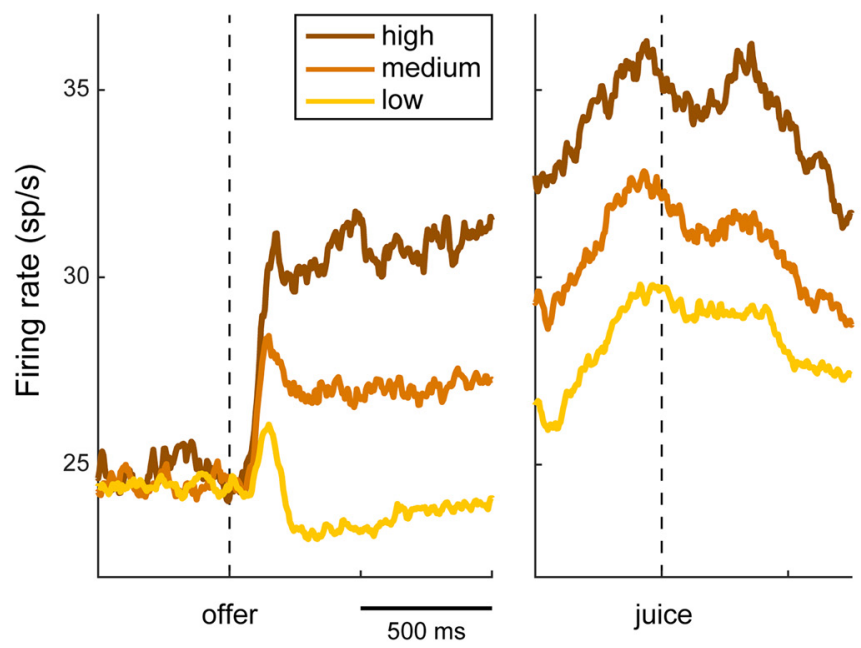

C offer value, slope $>0$ (55 cells)

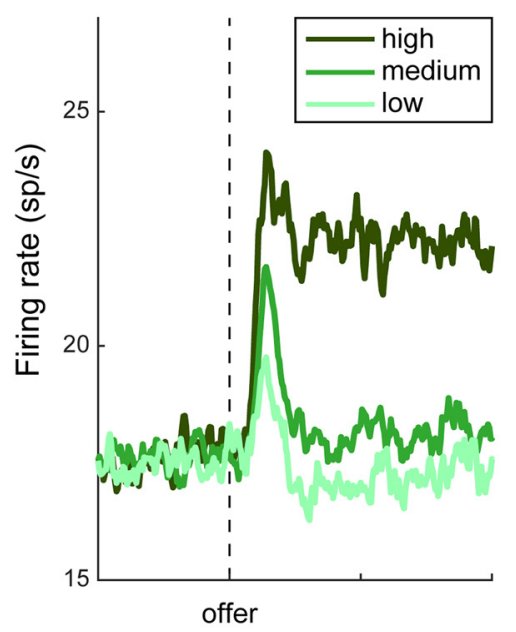

b chosen value, slope $<0$ ( 38 cells)
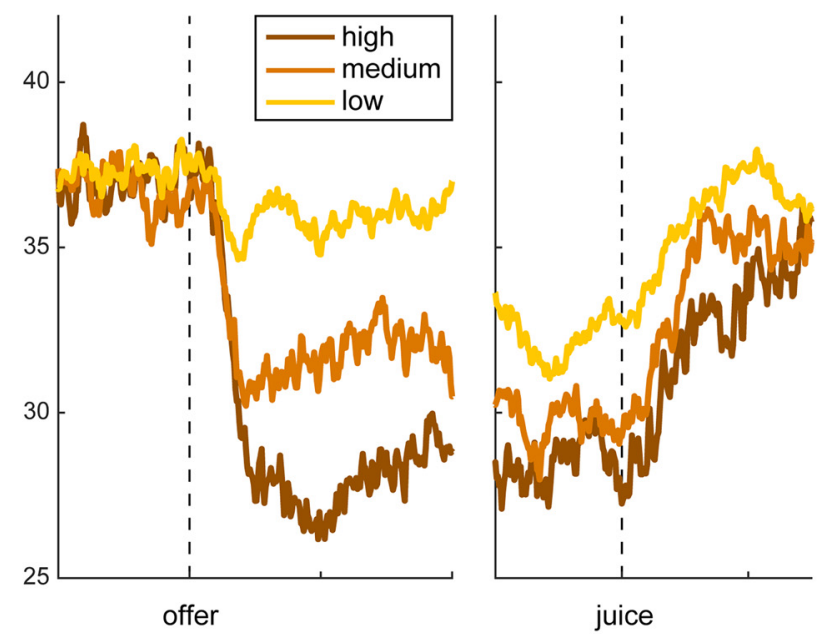

d chosen juice ( 35 cells)

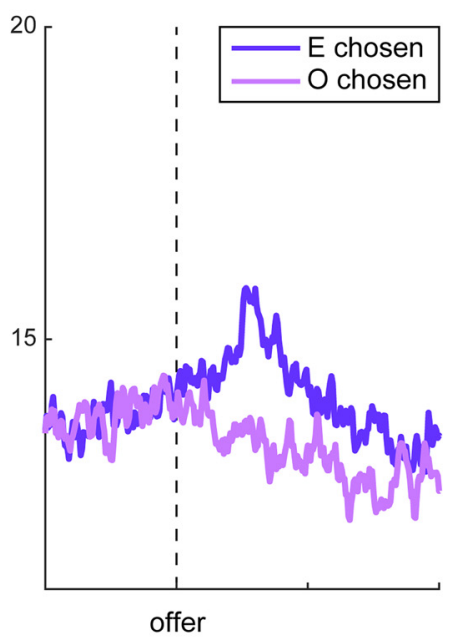

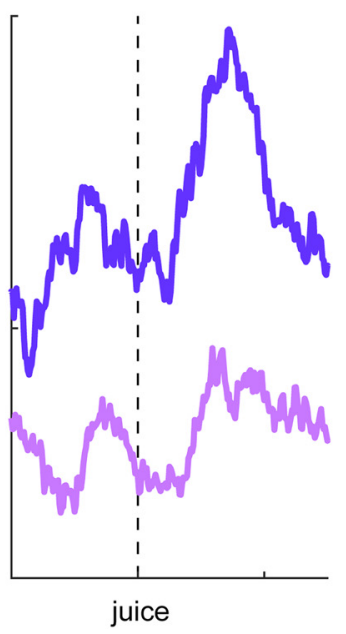

Figure 8. Population activity profiles in the BLA. $\boldsymbol{a}, \boldsymbol{b}$, Activity profiles for the population of chosen value cells with positive encoding $(\boldsymbol{a})$ and negative encoding ( $\boldsymbol{b})$. To calculate activity profiles, trials were separately aligned at offer and juice delivery. Trials were divided into three groups according to the chosen value. For each trial, the spike train was smoothed using a kernel that mimicked the postsynaptic potential by exerting influence only forward in time (decay time constant, $20 \mathrm{~ms}$ ). Resulting traces were averaged across trials and across cells. The three traces refer to low, medium, and high chosen values. $\boldsymbol{c}$, Activity profile for the population of offer value cells with positive encoding. The three traces refer to low, medium, and high values of the encoded juice. $\boldsymbol{d}$. Activity profile of the population of chosen juice cells. The two traces correspond to trials in which the animal chose the encoded juice or the other juice.

Table 4. Cell classes in the BLA and OFC

\begin{tabular}{lcc}
\hline & BLA & OFC \\
\hline Total & 1132 & 716 \\
Not task-related & 734 & 485 \\
Task-related & 398 & 231 \\
Offer value + & $55(15)$ & $61(32.5)$ \\
Offer value - & $16(4)$ & $5(3)$ \\
Chosen value + & $214(60)$ & $66(35)$ \\
Chosen value - & $38(11)$ & $27(14)$ \\
Chosen juice & $35(10)$ & $29(15.5)$ \\
Nonclassified & 40 & 43 \\
\hline
\end{tabular}

Numbers are cell counts; numbers in parentheses indicate the corresponding percentage of classified cells.

OFC-namely, offer value, chosen value, and chosen juice (Table 4 ). We then examined the activity profiles for the various populations, dividing trials into tertiles for offer value cells and chosen value cells and according to the choice outcome for chosen juice cells.

At the population level, the picture emerging from the new OFC data (Fig. 9) closely resembled that described previously
(Padoa-Schioppa, 2013). In particular, both chosen value cells (Fig. 9a) and offer value cells (Fig. 9c) presented a largely phasic modulation that emerged $\sim 200 \mathrm{~ms}$ after the offer and mostly dissipated within 500 ms. Conversely, chosen juice cells (Fig. 9d) revealed the choice outcome starting $\sim 250 \mathrm{~ms}$ after the offer and throughout the rest of the trial.

Comparing the two areas, one notable difference was that firing rates in the BLA were generally higher than in the OFC. For example, the preoffer activity (baseline) of chosen value cells with positive encoding was $\sim 25$ spikes/s (sp/s) in the BLA (Fig. $8 a$ ) and $<10 \mathrm{sp} / \mathrm{s}$ in the OFC (Fig. 9a). Similarly, the preoffer activity of offer value cells with positive encoding was $\sim 17 \mathrm{sp} / \mathrm{s}$ in the BLA (Fig. $8 c$ ) and $\sim 7 \mathrm{sp} / \mathrm{s}$ in the OFC (Fig. 9c). Statistical analyses confirmed that the difference in baseline (preoffer activity) between the two areas was statistically significant at the population level. This result held true in each monkey (both $p<10^{-20}$, Kruskal-Wallis test; Fig. 10).

To further examine the encoding of decision variables over time and in the two areas, we analyzed firing rates in $250 \mathrm{~ms}$ 


\section{a chosen value, slope $>0$ ( 66 cells)}

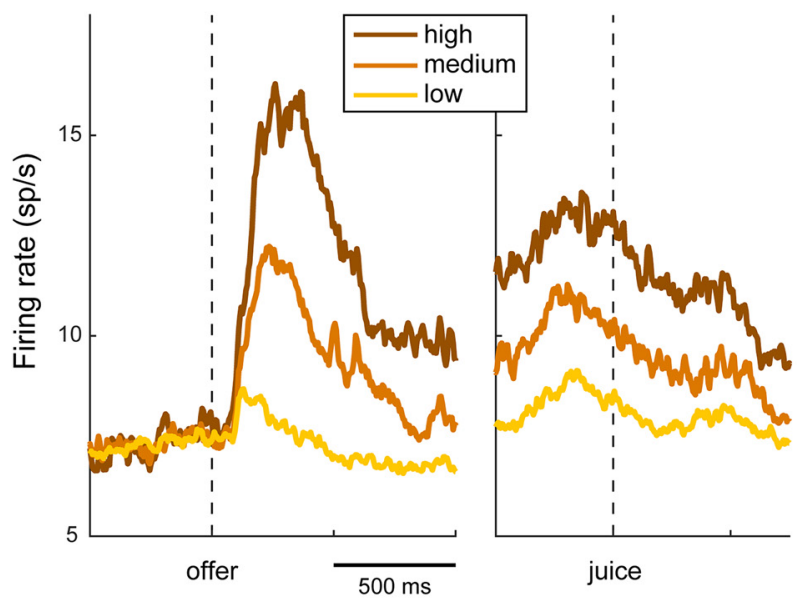

C offer value, slope $>0$ ( 61 cells)

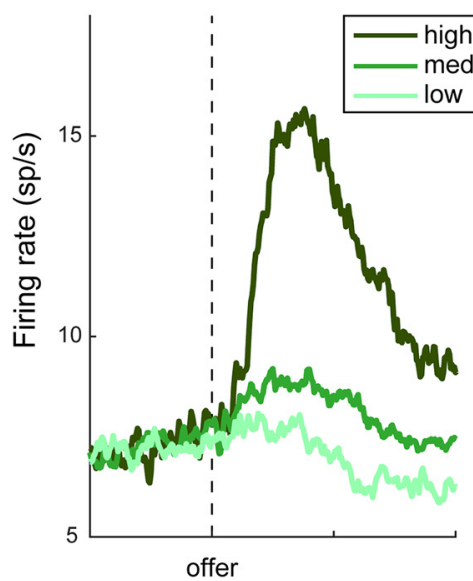

b

chosen value, slope $<0$ ( 27 cells)

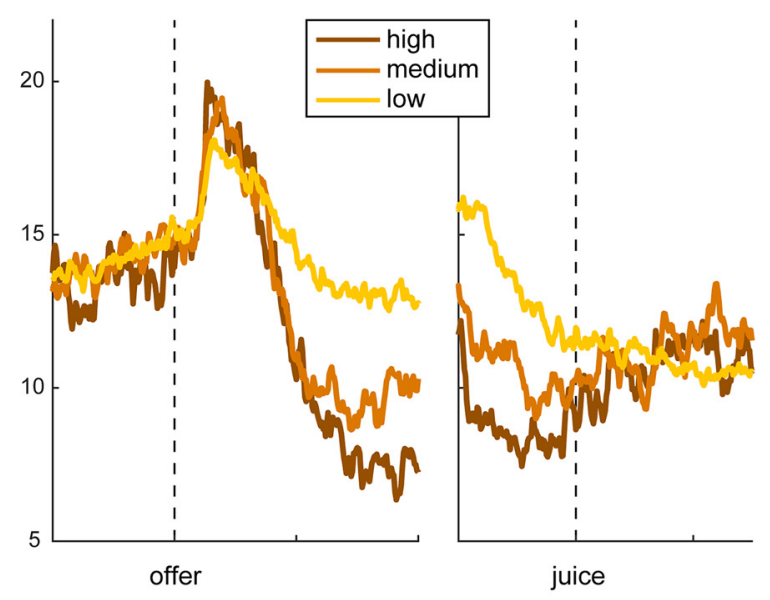

d chosen juice $(29$ cells)

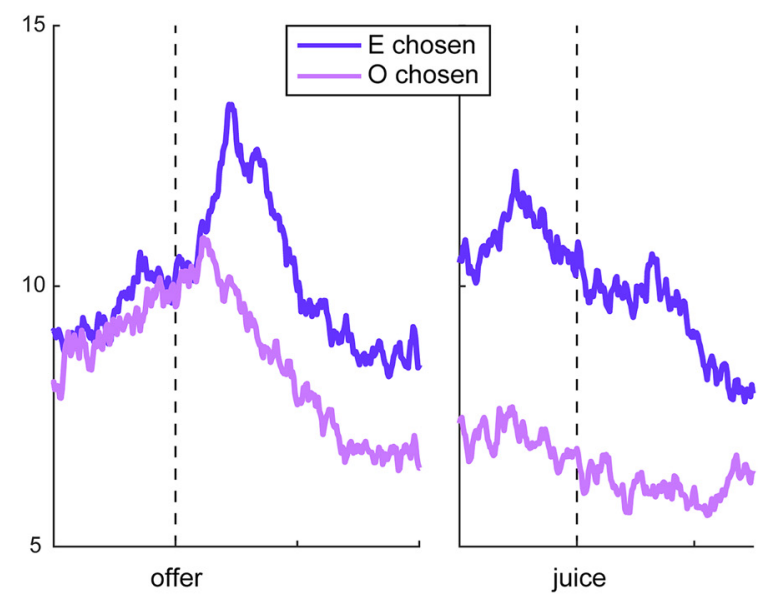

Figure 9. Population activity profiles in the OFC. Same format and conventions as in Figure 8. $\boldsymbol{a}$ - $\boldsymbol{d}$ Activity profiles for the populations of chosen value cells with positive encoding $(\boldsymbol{a})$, chosen value cells with negative encoding $(\boldsymbol{b})$, offer value cells with positive encoding (c) and chosen juice cells $(\boldsymbol{d})$.

sliding time windows shifted by $50 \mathrm{~ms}$. For each cell and each time window, we regressed the firing rate against the encoded variable. Each regression provided a slope and a $p$ value. We defined LP $=-\log _{10}(p)$, which essentially quantified the strength of the encoding. Figure 11 illustrates the results obtained for the two groups of cells (offer value, chosen value) with positive encoding in the two areas. For each neuron, we defined the tuning latency as the first time for which $p<0.01$. As illustrated in Figure 12, most tuning latencies were $<500 \mathrm{~ms}$.

Confirming the picture emerging from the population firing rates (Figs. 8, 9), it appeared that most chosen value cells in the BLA remained significantly tuned throughout the trial (Fig. 11b). In contrast, the tuning of most chosen value cells in the OFC appeared phasic (Fig. 11d). To quantify this effect, we focused on cells with a tuning latency of $\leq 250 \mathrm{~ms}$, and we assessed how many of them were still tuned $1 \mathrm{~s}$ after the offer onset. We found that the proportion of chosen value cells presenting sustained tuning was significantly higher in the BLA than in the OFC $\left(p=0.002, \chi^{2}\right.$ test). This trend held true in each animal ( $p=0.008$ and $p=$ 0.089). In contrast, no such difference across areas was found for offer value cells (all $p>0.5, \chi^{2}$ test). In both the BLA and OFC, approximately equal numbers of offer value cells presented phasic versus sustained tuning.

To summarize, quantitative comparisons of the results obtained for BLA and OFC revealed several differences. First,

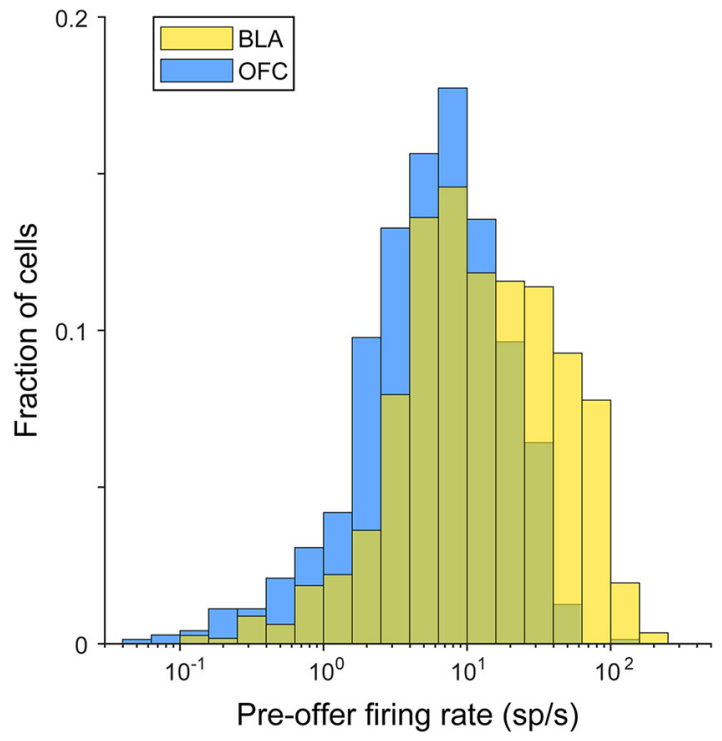

Figure 10. Distribution of baseline activity in the amygdala and OFC. Histograms show the distributions of mean firing rates recorded during the $500 \mathrm{~ms}$ preceding the offer (preoffer time window) in the BLA (yellow) and OFC (blue). Note that firing rates are plotted on a log scale. Green color indicates overlap between the two distributions. Across the population, the baseline activity recorded in the amygdala was significantly higher than that recorded in the OFC $\left(p<10^{-20}\right.$, Kruskal-Wallis test). This result held true in each animal (both $\left.p<10^{-20}\right)$. 
a

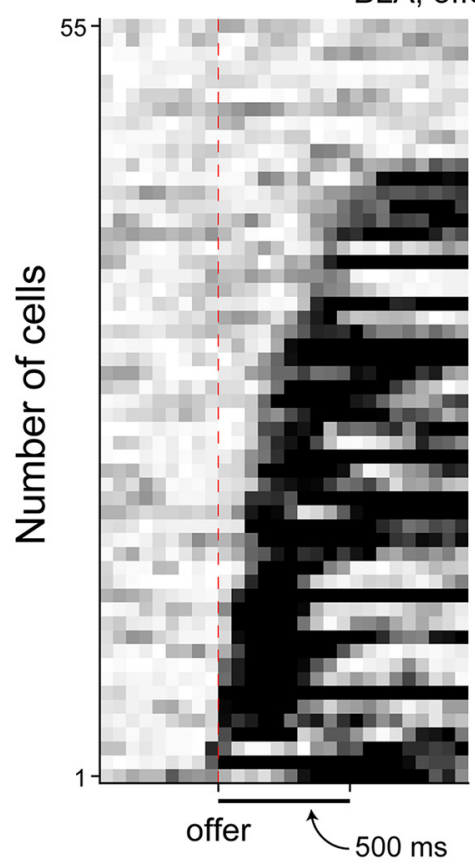

BLA, offer value

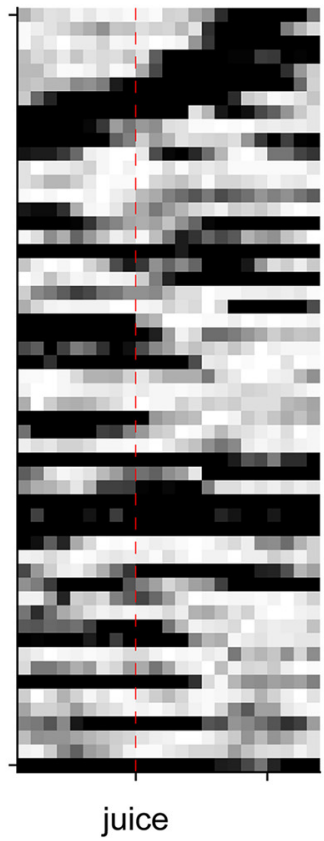

C

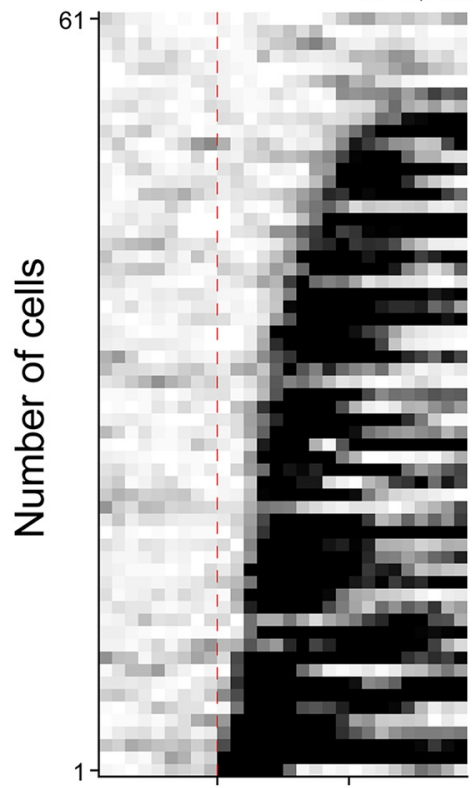

offer
OFC, offer value

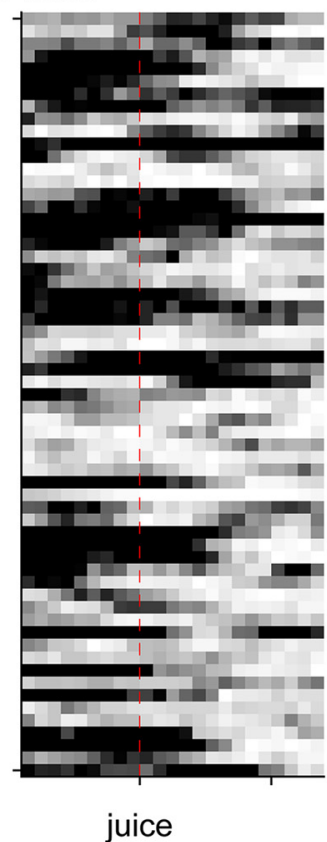

b

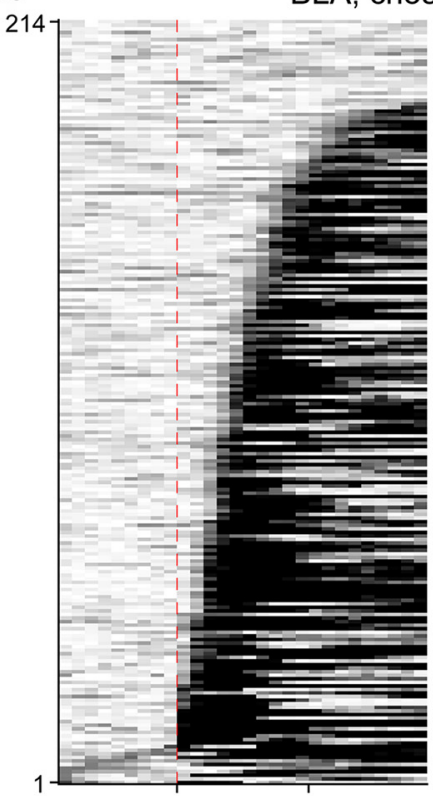

offer

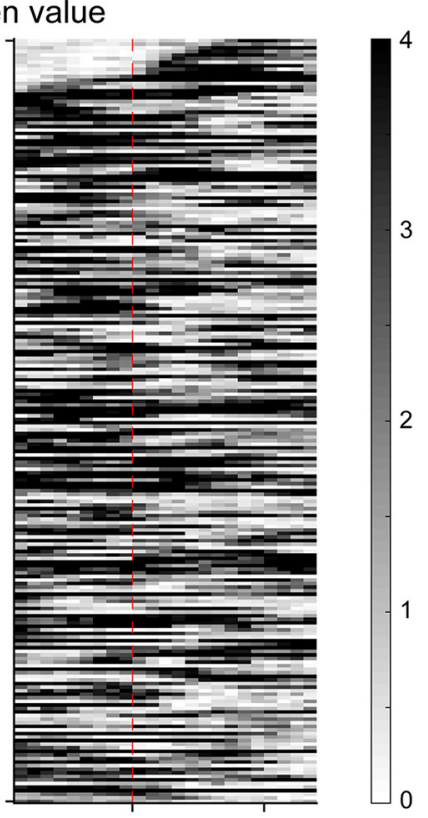

juice d

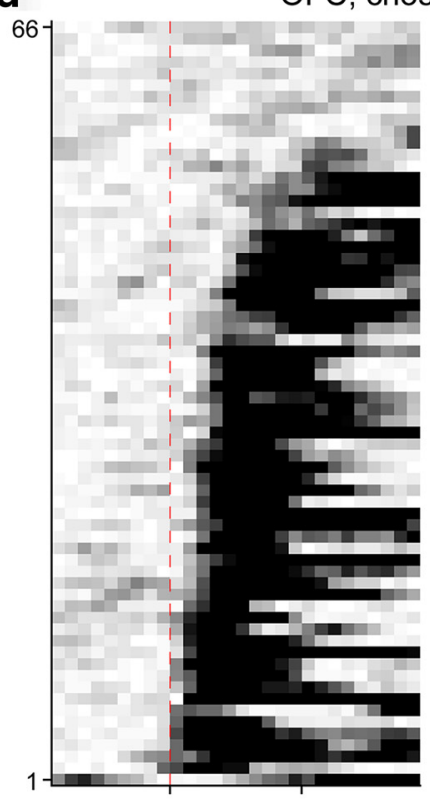

offer

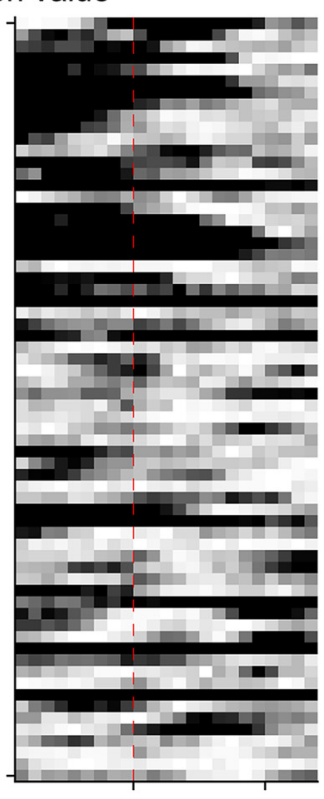

juice

Figure 11. Neuronal encoding of decision variables, temporal profile. $a$, Amygdala, offer value cells $(N=55)$. Each cell was examined in $250 \mathrm{~ms} \mathrm{sliding} \mathrm{time} \mathrm{windows} \mathrm{shifted} \mathrm{by} 50 \mathrm{~ms}$. Time windows were separately aligned with offer onset (left) and juice delivery (right). In each window, a linear regression of firing rates against the offer value provided a $p$ value for the regression slope. The smaller the $p$ value was, the stronger the encoding was. For each cell and for each time window, we computed $L P=-\log _{10}(p)$. The panel shows, for each cell, $L P$ over time. To interpret gray shades, see the legend on the right. Note that $L P=0,2$, and 4 correspond to $p=1, p=0.01$, and $p=10^{-4}$, respectively. In the plot, cells were ranked from bottom to top according to the tuning latency. $\boldsymbol{b}$, Amygdala, chosen value cells $(N=214)$. $\boldsymbol{c}$, OFC, offer value cells $(N=61)$. $\boldsymbol{d}, 0 \mathrm{OFC}$, chosen value cells $(N=66)$. $\boldsymbol{b}-\boldsymbol{d}$, All conventions are as in $\boldsymbol{a}$.

mean firing rates were overall higher in the BLA than in the OFC (Fig. 10). Second, the proportion of task-related cells encoding the chosen value was significantly larger in the BLA than in the OFC (BLA, 252/398 = 64\%; OFC, 93/231 = 41\%; Pearson's $\chi^{2}$ test; $\left.p<10^{-5}\right)$. The higher prevalence of chosen value cells in the BLA compared with the OFC held true even when we considered separately the data for the two animals $\left(p<10^{-4}\right.$, Mantel-Haenszel test). Third, the encoding of chosen value was more sustained in the amygdala compared with the OFC.

\section{Discussion}

Economic choice behavior entails the computation and comparison of subjective values. A large number of studies link these mental processes to the OFC. In contrast, the role of other areas remains uncertain. In this respect, arguably the strongest candi- 

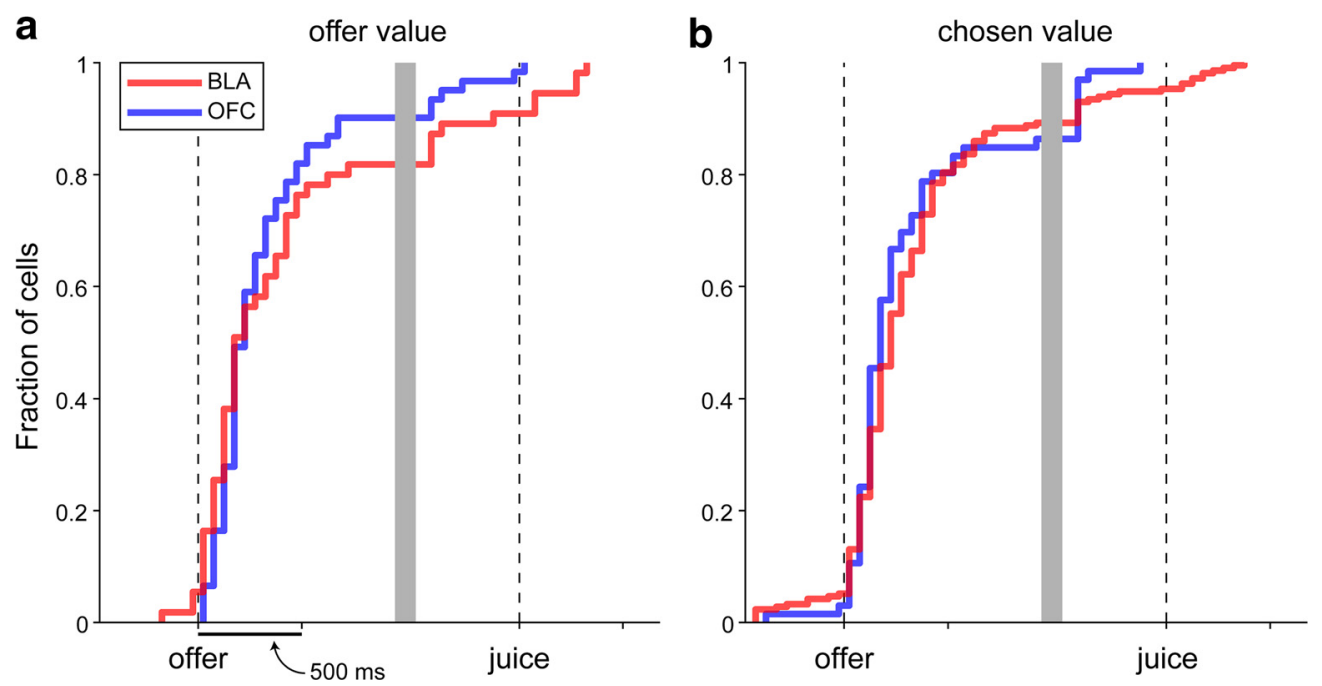

Figure 12. Tuning latencies in the amygdala and OFC. $\boldsymbol{a}$, Offer value cells. $\boldsymbol{b}$, Chosen value cells. In each panel, the results for the BLA and OFC are illustrated in red and blue, respectively.

date is the amygdala. Together with the central OFC (Gallagher et al., 1999; Rudebeck et al., 2013) and the mediodorsal thalamus (Mitchell et al., 2007; Izquierdo and Murray, 2010), the amygdala is the only brain region where lesions affect performance in reinforcer devaluation tasks (Málková et al., 1997; Blundell et al., 2001; Setlow et al., 2002; Fiuzat et al., 2017; Hart and Izquierdo, 2017). Because values driving goal-directed behaviors are closely related to values driving economic choices (O'Doherty, 2014; Padoa-Schioppa and Schoenbaum, 2015), the known effects of amygdala lesions suggest that this area might also play a role in economic decisions. Supporting this notion, previous neurophysiology results are broadly consistent with a representation of value in the BLA (Schoenbaum et al., 1998, 2003; SugaseMiyamoto and Richmond, 2005; Paton et al., 2006; Belova et al., 2008; Bermudez and Schultz, 2010; Roesch et al., 2010; Jenison et al., 2011; Grabenhorst et al., 2012; Peck et al., 2013; Leathers and Olson, 2017). At the same time, most previous studies of the BLA did not involve economic choices and/or did not contrast neuronal activity in the BLA with that recorded in the OFC. To fill these important gaps, we examined the activity of neurons in the BLA while monkeys performed a juice choice task. Approximately one-third of cells were task-related. Of these, $90 \%$ encoded one of the three decision variables: offer value, chosen value, or chosen juice. As a caveat, the explanatory power of chosen juice was statistically indistinguishable from that of variable chosen value $A \mid B$.

Intriguingly, the variables identified in the BLA are the same previously identified in the OFC (Padoa-Schioppa and Assad, 2006). In contrast, neurons in the dorsal and ventral ACC were found to encode different sets of variables (Cai and PadoaSchioppa, 2012). Aside from the caveat mentioned above, the BLA and OFC appeared to differ in two important ways. First, the majority $-\sim 2 / 3$ - of task-related cells in the BLA encoded the chosen value. In contrast, here and in other studies, cell counts for the three groups of cells in the OFC were more balanced, with 1/3-1/2 encoding the chosen value (Padoa-Schioppa, 2013; Xie and Padoa-Schioppa, 2016). Second, the two areas differed for the time course of this signal. Specifically, chosen value cells in the BLA presented high firing rates and a sustained modulation throughout the trial. In contrast, chosen value cells in the OFC had lower firing rates, and their modulation was generally phasic. In this respect, current measures for the OFC replicate previous findings (Padoa-Schioppa, 2013).
The implications of our results are potentially profound. The proposal that economic decisions are generated in a neural circuit within the OFC was partly motivated by the presence of different groups of neurons in this area capturing both the input (offer value) and the output (chosen juice, chosen value) of the decision process (Padoa-Schioppa and Conen, 2017). The current results broaden this perspective, suggesting that neuronal populations in the BLA may play an active role in the decision. However, more work is necessary to test this hypothesis. For one, the preponderance of chosen value responses and the time course of activation-sustained throughout the trial and peaking after juice delivery-suggest a role of the BLA in reward expectation and possibly consummatory behavior, as opposed to decisionmaking per se. Moreover, the lines of evidence implicating the OFC in economic decisions include the correlation between choice variability and neuronal variability in each of the three groups of cells (Padoa-Schioppa and Conen, 2017), the fact that disrupting neuronal activity in the OFC disrupts choices $(\mathrm{Ku}-$ wabara et al., unpublished observations), and the fact that one can predictably bias choices through low-current electrical stimulation (Ballesta et al., unpublished observations). Similar experiments should be conducted on the amygdala to ascertain its possible role in economic decisions.

Aside from whether or how BLA participates in the decision process, the fact that neurons in this area encode subjective values deserves some comment. Numerous previous results are consistent with a neuronal representation of economic value in this area (Kahn et al., 2002; Gottfried et al., 2003; Schoenbaum et al., 2003; Belova et al., 2008; Bermudez and Schultz, 2010; Jenison et al., 2011; Peck et al., 2013; Leathers and Olson, 2017). However, the behavioral paradigms used in most previous studies did not provide an operational measure of value. Thus, previous work did not examine whether contextual variability in subjective values measured behaviorally was matched at the neuronal level. In this respect, the present results are noteworthy. In particular, the identity between the neuronal and behavioral measure of relative values demonstrates that neurons in the BLA reflect the subjective nature of values as opposed to any physical property of the goods. Broadening the perspective, neuronal signals encoding the chosen value have been found in numerous brain regions, including the OFC (Padoa-Schioppa and Assad, 2006; Sul et al., 2010), dorsolateral and ventrolateral prefrontal cortex (Cai and Padoa- 
Schioppa, 2014), ventromedial prefrontal cortex (Strait et al., 2014), pregenual cingulate cortex (Amemori and Graybiel, 2012), dorsal and ventral ACC (Cai and Padoa-Schioppa, 2012; Hosokawa et al., 2013), and dorsal and ventral striatum (Lau and Glimcher, 2008; Cai et al., 2011). It may be noted that only a few studies have conclusively shown that neuronal signals reflected the subjective nature of values. With this caveat, the present results extend previous findings and contribute to the understanding that value signals are widely distributed and likely inform a large number of neural and cognitive processes.

\section{References}

Amaral DG, Price JL (1984) Amygdalo-cortical projections in the monkey (Macaca fascicularis). J Comp Neurol 230:465-496.

Amemori K, Graybiel AM (2012) Localized microstimulation of primate pregenual cingulate cortex induces negative decision-making. Nat Neurosci 15:776-785.

Balleine BW, Killcross AS, Dickinson A (2003) The effect of lesions of the basolateral amygdala on instrumental conditioning. J Neurosci 23:666-675.

Belova MA, Paton JJ, Salzman CD (2008) Moment-to-moment tracking of state value in the amygdala. J Neurosci 28:10023-10030.

Bermudez MA, Schultz W (2010) Reward magnitude coding in primate amygdala neurons. J Neurophysiol 104:3424-3432.

Blundell P, Hall G, Killcross S (2001) Lesions of the basolateral amygdala disrupt selective aspects of reinforcer representation in rats. J Neurosci 21:9018-9026.

Cai X, Padoa-Schioppa C (2012) Neuronal encoding of subjective value in dorsal and ventral anterior cingulate cortex. J Neurosci 32:3791-3808.

Cai X, Padoa-Schioppa C (2014) Contributions of orbitofrontal and lateral prefrontal cortices to economic choice and the good-to-action transformation. Neuron 81:1140-1151.

Cai X, Kim S, Lee D (2011) Heterogeneous coding of temporally discounted values in the dorsal and ventral striatum during intertemporal choice. Neuron 69:170-182.

Chau BK, Sallet J, Papageorgiou GK, Noonan MP, Bell AH, Walton ME, Rushworth MF (2015) Contrasting roles for orbitofrontal cortex and amygdala in credit assignment and learning in macaques. Neuron 87:1106-1118.

Cho YT, Ernst M, Fudge JL (2013) Cortico-amygdala-striatal circuits are organized as hierarchical subsystems through the primate amygdala. J Neurosci 33:14017-14030.

Corbit LH, Balleine BW (2005) Double dissociation of basolateral and central amygdala lesions on the general and outcome-specific forms of pavlovian-instrumental transfer. J Neurosci 25:962-970.

Costa VD, Dal Monte O, Lucas DR, Murray EA, Averbeck BB (2016) Amygdala and ventral striatum make distinct contributions to reinforcement learning. Neuron 92:505-517.

De Martino B, Camerer CF, Adolphs R (2010) Amygdala damage eliminates monetary loss aversion. Proc Natl Acad Sci U S A 107:3788-3792.

Dunn OJ, Clark V (1987) Applied statistics: analysis of variance and regression, Ed 2. New York: Wiley.

Fiuzat EC, Rhodes SE, Murray EA (2017) The role of orbitofrontalamygdala interactions in updating action-outcome valuations in macaques. J Neurosci 37:2463-2470.

Gallagher M, McMahan RW, Schoenbaum G (1999) Orbitofrontal cortex and representation of incentive value in associative learning. J Neurosci 19:6610-6614.

Garavan H, Pendergrass JC, Ross TJ, Stein EA, Risinger RC (2001) Amygdala response to both positively and negatively valenced stimuli. Neuroreport 12:2779-2783.

Ghashghaei HT, Barbas H (2002) Pathways for emotion: interactions of prefrontal and anterior temporal pathways in the amygdala of the rhesus monkey. Neuroscience 115:1261-1279.

Glantz SA, Slinker BK (2001) Primer of applied regression and analysis of variance, Ed 2. New York: McGraw-Hill.

Gottfried JA, O’Doherty J, Dolan RJ (2003) Encoding predictive reward value in human amygdala and orbitofrontal cortex. Science 301:1104-1107.

Grabenhorst F, Hernádi I, Schultz W (2012) Prediction of economic choice by primate amygdala neurons. Proc Natl Acad Sci U S A 109:18950-18955.

Grabenhorst F, Hernadi I, Schultz W (2016) Primate amygdala neurons evaluate the progress of self-defined economic choice sequences. Elife 5:e18731.
Hart EE, Izquierdo A (2017) Basolateral amygdala supports the maintenance of value and effortful choice of a preferred option. Eur J Neurosci 45:388-397.

Hosokawa T, Kennerley SW, Sloan J, Wallis JD (2013) Single-neuron mechanisms underlying cost-benefit analysis in frontal cortex. J Neurosci 33:17385-17397.

Izquierdo A, Murray EA (2010) Functional interaction of medial mediodorsal thalamic nucleus but not nucleus accumbens with amygdala and orbital prefrontal cortex is essential for adaptive response selection after reinforcer devaluation. J Neurosci 30:661-669.

Jenison RL, Rangel A, Oya H, Kawasaki H, Howard MA (2011) Value encoding in single neurons in the human amygdala during decision making. J Neurosci 31:331-338.

Kahn I, Yeshurun Y, Rotshtein P, Fried I, Ben-Bashat D, Hendler T (2002) The role of the amygdala in signaling prospective outcome of choice. Neuron 33:983-994.

Lau B, Glimcher PW (2008) Value representations in the primate striatum during matching behavior. Neuron 58:451-463.

Leathers ML, Olson CR (2017) In monkeys making value-based decisions, amygdala neurons are sensitive to cue value as distinct from cue salience. J Neurophysiol 117:1499-1511.

Málková L, Gaffan D, Murray EA (1997) Excitotoxic lesions of the amygdala fail to produce impairment in visual learning for auditory secondary reinforcement but interfere with reinforcer devaluation effects in rhesus monkeys. J Neurosci 17:6011-6020.

Mitchell AS, Browning PG, Baxter MG (2007) Neurotoxic lesions of the medial mediodorsal nucleus of the thalamus disrupt reinforcer devaluation effects in rhesus monkeys. J Neurosci 27:11289-11295.

Morecraft RJ, Geula C, Mesulam MM (1992) Cytoarchitecture and neural afferents of orbitofrontal cortex in the brain of the monkey. J Comp Neurol 323:341-358.

O’Doherty JP (2014) The problem with value. Neurosci Biobehav Rev 43:259-268.

Onken A, Xie J, Panzeri S, Padoa-Schioppa C (2019) Categorical encoding of decision variables in orbitofrontal cortex. PLoS Comput Biol 15:e1006667.

Ono T, Nishijo H, Uwano T (1995) Amygdala role in conditioned associative learning. Prog Neurobiol 46:401-422.

Padoa-Schioppa C (2013) Neuronal origins of choice variability in economic decisions. Neuron 80:1322-1336.

Padoa-Schioppa C, Assad JA (2006) Neurons in orbitofrontal cortex encode economic value. Nature 441:223-226.

Padoa-Schioppa C, Conen KE (2017) Orbitofrontal cortex: a neural circuit for economic decisions. Neuron 96:736-754.

Padoa-Schioppa C, Schoenbaum G (2015) Dialogue on economic choice, learning theory, and neuronal representations. Curr Opin Behav Sci 5:16-23.

Parkinson JA, Crofts HS, McGuigan M, Tomic DL, Everitt BJ, Roberts AC (2001) The role of the primate amygdala in conditioned reinforcement. J Neurosci 21:7770-7780.

Paton JJ, Belova MA, Morrison SE, Salzman CD (2006) The primate amygdala represents the positive and negative value of visual stimuli during learning. Nature 439:865-870.

Peck CJ, Lau B, Salzman CD (2013) The primate amygdala combines information about space and value. Nat Neurosci 16:340-348.

Porrino LJ, Crane AM, Goldman-Rakic PS (1981) Direct and indirect pathways from the amygdala to the frontal lobe in rhesus monkeys. J Comp Neurol 198:121-136.

Roesch MR, Calu DJ, Esber GR, Schoenbaum G (2010) Neural correlates of variations in event processing during learning in basolateral amygdala. J Neurosci 30:2464-2471.

Rudebeck PH, Saunders RC, Prescott AT, Chau LS, Murray EA (2013) Prefrontal mechanisms of behavioral flexibility, emotion regulation and value updating. Nat Neurosci 16:1140-1145.

Saez RA, Saez A, Paton JJ, Lau B, Salzman CD (2017) Distinct roles for the amygdala and orbitofrontal cortex in representing the relative amount of expected reward. Neuron 95:70-77.e3.

Schoenbaum G, Chiba AA, Gallagher M (1998) Orbitofrontal cortex and basolateral amygdala encode expected outcomes during learning. Nat Neurosci 1:155-159.

Schoenbaum G, Setlow B, Saddoris MP, Gallagher M (2003) Encoding predicted outcome and acquired value in orbitofrontal cortex during 
cue sampling depends upon input from basolateral amygdala. Neuron 39:855-867.

Setlow B, Gallagher M, Holland PC (2002) The basolateral complex of the amygdala is necessary for acquisition but not expression of CS motivational value in appetitive pavlovian second-order conditioning. Eur J Neurosci 15:1841-1853.

Strait CE, Blanchard TC, Hayden BY (2014) Reward value comparison via mutual inhibition in ventromedial prefrontal cortex. Neuron 82: $1357-1366$.

Sugase-Miyamoto Y, Richmond BJ (2005) Neuronal signals in the monkey basolateral amygdala during reward schedules. J Neurosci 25:11071-11083.

Sul JH, Kim H, Huh N, Lee D, Jung MW (2010) Distinct roles of rodent orbitofrontal and medial prefrontal cortex in decision making. Neuron 66:449-460.

Uwano T, Nishijo H, Ono T, Tamura R (1995) Neuronal responsiveness to various sensory stimuli, and associative learning in the rat amygdala. Neuroscience 68:339-361.

van Honk J, Eisenegger C, Terburg D, Stein DJ, Morgan B (2013) Generous economic investments after basolateral amygdala damage. Proc Natl Acad Sci U S A 110:2506-2510.

Wallis JD (2011) Cross-species studies of orbitofrontal cortex and valuebased decision-making. Nat Neurosci 15:13-19.

Xie J, Padoa-Schioppa C (2016) Neuronal remapping and circuit persistence in economic decisions. Nat Neurosci 19:855-861.

Zangemeister L, Grabenhorst F, Schultz W (2016) Neural basis for economic saving strategies in human amygdala-prefrontal reward circuits. Curr Biol 26:3004-3013.

Zhang W, Schneider DM, Belova MA, Morrison SE, Paton JJ, Salzman CD (2013) Functional circuits and anatomical distribution of response properties in the primate amygdala. J Neurosci 33:722-733. 